\title{
Canonical quantization of the string with dynamical geometry and anomaly free nontrivial string in two dimensions
}

\author{
M.O.Katanaev * \\ Steklov Mathematical Institute, \\ Vavilov St., 42, Moscow, 117966, Russia \\ E-mail: katanaev@mi.ras.ru
}

6 October 1993

\begin{abstract}
Hamiltonian formulation of the string with dynamical geometry and two-dimensional gravity with torsion is given. Canonical Hamiltonian equals to the linear combination of first class constraints satisfying closed algebra. It is the semidirect sum of the Virasoro algebra and the abelian subalgebra corresponding to the local Lorentz rotation. After making the canonical transformation the theory is quantized. It is proved that there exists Fock space representation of pure twodimensional gravity with torsion containing no central charge in the Virasoro algebra. Also constructed is the new Fock representation of a standard bosonic string. It is shown that two-dimensional string with dynamical geometry is anomaly free and describes two physical degrees of freedom.
\end{abstract}

\section{Introduction}

One of the most interesting model of contemporary physics is the bosonic string described by an action proportional to the area of the string world sheet (for a review see [1, 2] ). At the quantum level there arises the notion of critical dimension $D=26$ of the space-time where the theory can be consistently quantized. This is a drawback when the theory is considered as describing 4 dimensional extended objects. Bosonic string also contains a tachyon in its spectrum and therefore various modifications of the Lagrangian have been proposed last years.

The simplest geometric generalization of the bosonic string is the string with dynamical geometry that is obtained by adding the Lagrangian of two-dimensional gravity with torsion [3, 4]. The latter equals to the sum of curvature squared term, torsion squared term, and cosmological constant, the geometrical quantities being constructed from twodimensional metric and torsion of the string world sheet. On the other hand this model

*Supported by Russian fund of fundamental investigations RFFI-93-011-140 
can be viewed as two-dimensional gravity and $D$ minimally coupled massless scalar fields corresponding to the string coordinates.

At the same time two-dimensional gravity with torsion is of interest by itself at least for two reasons. Firstly, it provides highly nontrivial gravity model in two dimensions with well defined action. Therefore its elucidation will shed a new light on the whole problem of quantum gravity. Secondly, it has direct physical application in the theory of solids because in Euclidean space it describes the membranes with dislocations and disclinations (see [5] and references there in).

Recently there was a progress in two-dimensional gravity with torsion. The highly nonlinear equations of motion turn out to possess the explicit general local solution which have been found in the conformal gauge [6], the light-cone gauge [7], and in the gauge where torsion components play the role of space-time coordinates [8, 9]. Earlier there were found an instantonlike [10] and all static solutions [4]. It is interesting that the Liouville model (constant curvature and zero torsion) is incorporated as one of the two sectors of two-dimensional gravity with torsion. In the space-time equipped with general type metric and torsion satisfying the equations of motion of two-dimensional gravity with torsion, all extremals and geodesics have been found and analysed in the conformal gauge [11]. In light-cone gauge part of them have been analysed also in Ref.[7]. It is worth attention that two-dimensional gravity with torsion admits not only local but also a global analysis. Recently all global maximally continued solutions have been found and classified up to an action of the descrete transformation group [12]. This means that at the classical level the theory admits complete local as well as global analysis. Among the global solutions there are solutions describing black and white hole configurations which are similar to the Kruskal extension of the Schwartzschild solution. The knowledge of all global solutions of the model yields the hope to investigate in detail the relevance of global structure of gravity to quantization.

For quantization of the model it is very important to formulate the theory in Hamiltonian form. Two-dimensional gravity with torsion was canonically formulated in Refs. [13, 14. (Earlier it was canonically formulated only in the conformal gauge [15].) In Ref. 113 the lightcone variable plays the role of time. The theory is proved to have 6 first-class costraints. The authors show that 3 of them together with the momenta form the quadratically deformed iso(2,1) algebra which is interpreted as the novel symmetry of the model. In Ref. [14] the theory is canonically formulated, a timelike coordinate playing the role of time variable. The results are in agreement with [13. In Ref. [16] the Hamiltonian formulation was also considered.

Both Lagrangian and Hamiltonian formulations show that pure two-dimensional gravity with torsion describes no dynamical continuous degree of freedom. This statement will be surely untrue if one adds the matter fields to the theory. This is one of the reasons why we treat the string with dynamical geometry in the present paper. Its canonical formulation was not considered earlier.

In the present paper the string with dynamical geometry is written in Hamiltonian form. When one transforms a complicated nonlinear theory with local invariance from the Lagrangian to the Hamiltonian form the choice of independent variables become of prime importance. In contrast to Refs. [13, 14] we choose to work with the Arnowitt, Deser, Misner [17] decomposition of the two-dimensional metric and the corresponding parametrization of the zweibein. This considerably simplifies calculations. We found six first-class constraints satisfying closed algebra. It is the direct sum of 3-dimensional 
abelian algebra of primary constraints and the new 3-dimensional algebra of secondary constraints that is the semidirect sum of the one-dimensional abelian subalgebra and the Virasoro algebra. This is in agreement with the following general consideration. We know that the Virasoro algebra is connected with the conformal symmetry which is the part of general coordinate invariance. In our case the model is also invariant under 1-parameter local Lorentz rotation. The last symmetry yields the nontrivial generalization of the Virasoro algebra.

The Hamiltonian form of the theory shows that the string with dynamical geometry describes $D$ dynamical degrees of freedom. We have found restrictions on the coupling constants yielding positive definite canonical Hamiltonian for all physical modes. To elucidate the quantum constraint algebra it is very useful to make the nonlocal canonical transformation corresponding to the decomposition of the zweibein into longitudinal and transversal part. In fact the longitudinal component coinsides with the conformal piece of the metric while the transversal one coinsides with the Lorentz angle and is unphysical.

The theory is canonically quantized without any approximation. That is for coordinates and momenta we introduce creation and annihilation operators, construct the Fock space and prove that the normally ordered constraints of pure two-dimensional gravity with torsion satisfy the Virasoro algebra without central charge. We have also found new Fock representation for an ordinary bosonic string where the central charge differs by two from its standard value. This means that an anomaly canarise only due to the ghost fields which are not considered in the present paper. This is a new quantum model describing two physical continuous degrees of freedom: the first is the space component of the string and the second is the conformal piece of the metric of the string world sheet.

There are other approaches to quantization of pure two-dimensional gravity with torsion. In Refs. [14, 18] the theory is canonically quantized using the symmetric representation in coordinates and momenta of Hermitian operators. An important progress have been done within the path integral approach. The model was shown to be renormalisable [19] and integrable at the quantum level [20]. The Lagrangian BRST quantization of the model was considered in [21]. There the action of two-dimensional gravity with torsion was written in the first order form which can be considered as a gauge theory of quadratically deformed two-dimensional Poincaré algebra [22].

The paper is devided in two parts having subsections. In the first part we discuss the classical canonical formulation of the theory. The second part is devoted to the quantum aspects of the theory.

\section{Hamiltonian formulation}

If one tries to quantize a theory nonperturbatively then the canonical formulation seems to be the most adequate starting point. This stems from the fact that nonlinearity of a theory does not yield any principle difficulty when a Lagrangian theory is transformed into a Hamiltonian form. And then it can be canonically quantized replacing Poisson brackets by commutators. There is also not much freedom in the introduction of creation and annihilation operators, constructing a Fock space representation of any nonlinear quantum field theory. There will be no approximation. The main problem arising in such approach to gauge theories is the investigation of an algebra of quantum operators which at the classical level satisfy some algebra corresponding to gauge invariance. The aim 
here is the construction of such representation in which the quantum algebra coincides with the Poisson bracket algebra. In this case we say that the theory contains no anomaly (here we neglect a possible contribution from ghosts fields).

At the classical level a canonically formulated theory has a wide class of equivalent realisations connected by (in general nonlinear) canonical transformations. These realisations can yield unitary inequivalent representations of the quantum theory. For example, everybody knows how to quantize a harmonic oscillator, but if one makes a nonlinear canonical transformation then the resulting quantum theory may be disastrous and unitary inequivalent to the standard realization. So the choice of canonical variables becomes of prime importance for nonlinear theories. I think the guiding principle here is the simplicity of the resulting Hamiltonian theory were the quantization can be performed consistently.

Situation in two-dimensional gravity with torsion is the following. Let us find even one canonical formulation where the quantization can be performed. This canonical formulation is given in the first part of the paper. Here we encount four canonical transformations and show that the resulting canonical theory for physical modes is similar to the Liouville theory interacting with the bosonic string. But it is more general and allows one to perform consistent quantization.

\subsection{The Lagrangian}

In this section we introduce notations, write down the Lagrangian and its symmetry transformations.

Let $X^{\mu}\left(\zeta^{\alpha}\right), \mu=0,1, \ldots, D-1$, be coordinates of the string moving in $D$-dimensional flat Minkowskian space-time equiped with the metric $\eta_{\mu \nu}=\operatorname{diag}(+-\ldots-)$. Here $\zeta^{\alpha}$, $\alpha=0,1$, are coordinates on the string world sheet. Their range will be spesified later, and at the moment we consider them as a local coordinate system. To give meaning of the Hamiltonian formulation we assume that tangent vector $\partial_{0} X^{\mu}$ is everywhere timelike, $\partial_{0} X_{\mu} \partial_{0} X^{\mu}>0$, and tangent vector $\partial_{1} X^{\mu}$ is everywhere spacelike, $\partial_{1} X_{\mu} \partial_{1} X^{\mu}<0$.

We assume that the world sheet of a string with dynamical geometry is equiped with the Riemann-Cartan geometry, that is the metric $g_{\alpha \beta}(\zeta)=g_{\beta \alpha}(\zeta)$ and the torsion $T_{\alpha \beta}^{\gamma}(\zeta)=-T_{\beta \alpha}^{\gamma}(\zeta)$ are given.

The action of the string with dynamical geometry has the form [3]

$$
I=\int d^{2} \zeta e\left(L_{X}+L_{G}\right), \quad e=\operatorname{det} e_{\alpha}^{a},
$$

where $e$ defines the volume measure. Lagrangian of the model equals to the sum of the standard Lagrangian of the bosonic string,

$$
L_{X}=-\frac{1}{2} \rho g^{\alpha \beta} \partial_{\alpha} X_{\mu} \partial_{\beta} X^{\mu}
$$

and the Lagrangian of two-dimensional gravity with torsion,

$$
L_{G}=\frac{1}{4} \gamma R_{\alpha \beta}^{a b} R_{a b}^{\alpha \beta}+\frac{1}{4} \beta T_{\alpha \beta}{ }^{a} T_{a}^{\alpha \beta}-\lambda,
$$

where $R_{\alpha \beta}^{a b}$ and $T_{\alpha \beta}{ }^{a}$ are intrinsic curvature and torsion of the string world sheet. Here $\rho$ is a mass density of the string, $\gamma$ and $\beta$ are coupling constants and $\lambda$ is a cosmological constant. Raising and lowering of Greek indices from the beginning of the alphabet is 
performed by using metric $g_{\alpha \beta}$ and its inverse $g^{\alpha \beta}$, while Latin indices are raised and lowered by using two-dimensional Minkowskian metric $\eta_{a b}=\operatorname{diag}(+-)$. Transformation of the Greek indices into the Latin ones and vice versa is performed by using zweibein field $e_{\alpha}{ }^{a}(\zeta), g_{\alpha \beta}=e_{\alpha}{ }^{a} e_{\beta}{ }^{b} \eta_{a b}$.

The zweibein $e_{\alpha}{ }^{a}$ and the Lorentz connection $\omega_{\alpha}^{a b}=-\omega_{\alpha}^{b a}$ are independent variables and in this case equations of motion are of second order. Curvature and torsion are expressed in these variables as follows

$$
\begin{aligned}
R_{\alpha \beta}{ }^{a b} & =\partial_{\alpha} \omega_{\beta}^{a b}-\omega_{\alpha}^{a c} \omega_{\beta c}^{b}-(\alpha \leftrightarrow \beta), \\
T_{\alpha \beta}{ }^{a} & =\partial_{\alpha} e_{\beta}{ }^{a}-\omega_{\alpha}^{a b} e_{\beta b}-(\alpha \leftrightarrow \beta) .
\end{aligned}
$$

In two dimensions one can always parametrize Lorentz connection by a pseudovector field $B_{\alpha}(\zeta)$

$$
\omega_{\alpha}^{a b}=B_{\alpha} \epsilon^{a b}, \quad \epsilon_{a b}=-\epsilon_{b a}, \epsilon_{01}=1 .
$$

Then curvature takes the form

$$
R_{\alpha \beta}^{a b}=F_{\alpha \beta} \epsilon^{a b}, \quad F_{\alpha \beta}=\partial_{\alpha} B_{\beta}-\partial_{\beta} B_{\alpha} .
$$

In two dimensions curvature tensor is completely defined by the scalar curvature $R$ and torsion tensor is defined by its trace $T_{a}$

$$
\begin{aligned}
R_{a b c d} & =-\frac{1}{2} \epsilon_{a b} \epsilon_{c d} R, \quad R=R_{a b}{ }^{a b}, \\
T_{a b}{ }^{c} & =\delta_{a}^{c} T_{b}-\delta_{b}^{c} T_{a}, \quad T_{b}=T_{a b}{ }^{a} .
\end{aligned}
$$

By construction the action (1) is invariant under general coordinate transformations and local Lorentz rotation. To clarify the meaning of the first class constraints in the following we need the explicit form of the infinitesimal symmetry transformations of the fields. Coordinate transformations with infinitesimal parameter $\delta \zeta^{\alpha}=\epsilon^{\alpha}(\zeta)$ look as follows

$$
\begin{aligned}
\delta X^{\mu} & =-\epsilon^{\beta} \partial_{\beta} X^{\mu}, \\
\delta e_{\alpha}{ }^{a} & =-\partial_{\alpha} \epsilon^{\beta} e_{\beta}{ }^{a}-\epsilon^{\beta} \partial_{\beta} e_{\alpha}{ }^{a}, \\
\delta e & =-\partial_{\alpha} \epsilon^{\alpha} e-\epsilon^{\alpha} \partial_{\alpha} e \\
\delta \omega_{\alpha}{ }^{a b} & =-\partial_{\alpha} \epsilon^{\beta} \omega_{\beta}^{a b}-\epsilon^{\beta} \partial_{\beta} \omega_{\alpha}^{a b}, \\
\delta B_{\alpha} & =-\partial_{\alpha} \epsilon^{\beta} B_{\beta}-\epsilon^{\beta} \partial_{\beta} B_{\alpha} .
\end{aligned}
$$

Under the Lorentz rotation by the angle $\omega^{a b}=-\omega^{b a}=\omega \epsilon^{a b}, \omega=-\frac{1}{2} \omega^{a b} \epsilon_{a b}$, the fields transform in the way

$$
\begin{aligned}
\delta X^{\mu} & =0 \\
\delta e_{\alpha}{ }^{a} & =\omega^{a}{ }_{b} e_{\alpha}{ }^{b} \\
\delta e & =0 \\
\delta \omega_{\alpha}{ }^{a}{ }_{b} & =\omega^{a}{ }_{c} \omega_{\alpha}{ }^{c}{ }_{b}-\omega_{\alpha}{ }^{a}{ }_{c} \omega^{c}{ }_{b}+\partial_{\alpha} \omega^{a}{ }_{b}, \\
\delta B_{\alpha} & =\partial_{\alpha} \omega .
\end{aligned}
$$

General coordinate transformations and local Lorentz rotation are parametrized by three arbitrary functions and are the only local symmetries of the string with dynamical geometry in the Lagrangian approach [14]. 


\subsection{Equations of motion and the limiting cases}

Before elucidating the general case corresponding to nonzero coupling constants in the action let us say a few words about the limiting cases. Varing the action (1) with respect to $X^{\mu}, B_{\alpha}$, and $e_{\alpha}{ }^{a}$ one obtains the following equations of motion

$$
\begin{aligned}
\nabla^{\alpha} \nabla_{\alpha} X^{\mu}-T^{\alpha} \nabla_{\alpha} X^{\mu} & =0, \\
\gamma \nabla_{\alpha} R+\beta T_{\alpha} & =0, \\
-\beta\left(\nabla_{\alpha} T_{\beta}-\nabla_{\gamma} T^{\gamma} g_{\alpha \beta}+\frac{1}{2} T_{\gamma} T^{\gamma} g_{\alpha \beta}\right)-\frac{1}{4} \gamma R^{2} g_{\alpha \beta} & \\
+\rho\left(\nabla_{\alpha} X_{\mu} \nabla_{\beta} X^{\mu}-\frac{1}{2} \nabla_{\gamma} X_{\mu} \nabla^{\gamma} X^{\mu} g_{\alpha \beta}\right)-\lambda g_{\alpha \beta} & =0,
\end{aligned}
$$

where $\nabla_{\alpha}$ denotes the true covariant derivative that acts with the Lorentz connection $\omega_{\alpha}^{a b}$ on Latin indices and the corresponding metrical connection $\Gamma_{\alpha \beta}{ }^{\gamma}$ on the Greek indices from the beginning of the alphabet. Remember that the Greek indices from the middle of the alphabet simply enumerate $D$ string coordinates which are scalars under changes of the coordinates on the string world sheet.

The case $\rho=0$ corresponds to pure two-dimensional gravity with torsion. In what follows we assume that $\rho>0$. This choice of the sign ensure positivity of the contributions from the space string coordinates $X^{\mu}, \mu \neq 0$, to the canonical Hamiltonian.

For $\gamma=0$, equation (8) for the Lorentz connection implies the zero torsion condition $T_{\alpha \beta}{ }^{a}=0$. Then the cosmological constant must be zero $\lambda=0$ as follows from the trace of Eq.(9). So in this case equations of motion (7)-(9) are equivalent to the equations of motion for the ordinary bosonic string.

For $\beta=0$, trace of Eq.(9) yields the constant curvature equation

$$
R^{2}=-2 F_{\alpha \beta} F^{\alpha \beta}=-4 \lambda / \gamma .
$$

Then Eq.(8) is identically satisfied, and Eqs.(7), (9) reduce to the equations of motion for the ordinary string for any value of the cosmological constant. Note that in this case Eq.(9) defines metric $g_{\alpha \beta}$ as the one induced by the embedding $\xi^{\alpha} \rightarrow X^{\mu}$. So the constant curvature equation (10) must be considered with respect to the unknown Lorentz connection. In general, torsion will differ from zero.

The general solution of pure two-dimensional gravity with torsion contains the constant curvature and zero torsion sector (the Liouville model) [4, 6]

$$
R^{2}=-4 \lambda / \gamma, \quad T_{a b c}=0
$$

For this solution Eqs.(7)-(9) reduce to the ordinary equations for the bosonic string with one additional restriction on the metric. On the one hand Eq.(9) implies that the metric is induced by the embedding $\xi^{\alpha} \rightarrow X^{\mu}$. On the other hand the corresponding scalar curvature must be constant. That is Eq.(77) for string coordinates must be solved for constant curvature induced metric.

In the present paper definition of the coupling constants $\beta$ and $\gamma$ in Lagrangian (3) differs by the sign from that in my previous papers. This is done because in Section 2.8 we will prove that canonical Hamiltonian for all physical modes of the theory is positive definite if $\rho, \gamma, \beta>0$, and $\lambda \geq 0$. 


\subsection{Hamiltonian and constraints}

The Hamiltonian formulation of the Lagrangian model is the starting point for the canonical quantization procedure. For a given Lagrangian it is very important to choose the generalized coordinates in a suitable way. Different choices of the coordinates are related between themselves by the canonical transformations of the corresponding Hamiltonian systems, and are completely equivalent from the classical point of view. After several attempts I have found that more convinient is the Arnowitt, Deser, Misner like parametrization of the metric 17]

$$
g_{\alpha \beta}=g_{11}\left(\begin{array}{cc}
-N_{0}^{2}+N_{1}^{2} & N_{1} \\
N_{1} & 1
\end{array}\right),
$$

where $N_{0} \sqrt{-g_{11}}$ and $N_{1}$ are the lapse and shift functions. Later we will use the conformal gauge corresponding to $N_{0}=1$ and $N_{1}=0$. The inverse metric has the form

$$
g^{\alpha \beta}=\frac{1}{g_{11}}\left(\begin{array}{cc}
-\frac{1}{N_{0}^{2}} & \frac{N_{1}}{N_{0}^{2}} \\
\frac{N_{1}}{N_{0}^{2}} & 1-\frac{N_{1}^{2}}{N_{0}^{2}}
\end{array}\right),
$$

and $\operatorname{det} g_{\alpha \beta}=-N_{0}^{2} g_{11}^{2}$. In accordance with our choice of the signature we assume that $N_{0}>0$ and $g_{11}<0$.

The corresponding parametrization of the zweibein is

$$
\begin{aligned}
e_{0}{ }^{a} & =\left(N_{0} e_{1}{ }^{1}+N_{1} e_{1}{ }^{0}, N_{0} e_{1}{ }^{0}+N_{1} e_{1}{ }^{1}\right), \\
e_{1}{ }^{a} & =\left(e_{1}{ }^{0}, e_{1}{ }^{1}\right), \\
\operatorname{det} e_{\alpha}{ }^{a} & =-N_{0} e_{1}^{a} e_{1 a} .
\end{aligned}
$$

Therefore instead of $e_{\alpha}^{a}$ one can always choose four functions $N_{0}, N_{1}, e_{1}^{a}$ as the independent variables in the action (1). This is the first canonical transformation used in the present paper.

Let $X^{\mu}, N_{0}, N_{1}, e_{1}^{a}$, and $B_{\alpha}$ be the generalized coordinates in the phase space of the string with dynamical geometry. One readily sees that no time derivatives $\partial_{0} N_{0}, \partial_{0} N_{1}$, and $\partial_{0} B_{0}$ enter the Lagrangian. Therefore the corresponding conjugate momenta $P_{0}, P_{1}$, and $\pi^{0}$ equal zero, and we have three primary constraints

$$
P_{0} \approx P_{1} \approx \pi^{0} \approx 0
$$

where approximate equality means that the Poisson brackets should be calculated before solving the constraints. The nonzero conjugate momenta are

$$
\begin{aligned}
\Pi_{\mu} & =\frac{\delta L}{\delta\left(\partial_{0} X^{\mu}\right)}=-\rho e g^{0 \alpha} \partial_{\alpha} X_{\mu} \\
\pi_{a}^{1} & =\frac{\delta L}{\delta\left(\partial_{0} e_{1}{ }^{a}\right)}=\beta e T^{01}{ }_{a}=\beta \epsilon_{a b} T^{b}, \\
\pi^{1} & =\frac{\delta L}{\delta\left(\partial_{0} B_{1}\right)}=-2 \gamma e F^{01}=-\gamma R .
\end{aligned}
$$

We see that the theory contains 3 primary constraints. This is the minimal number because each local symmetry of the Lagrangian must be accompanied by one primary constraint 23]. 
After tedious but straightforward calculations one obtains the canonical Hamiltonian

$$
\begin{aligned}
H & =\int d \sigma\left(\Pi_{\mu} \partial_{0} X^{\mu}+\pi^{1}{ }_{a} \partial_{0} e_{1}^{a}+\pi^{1} \partial_{0} B_{1}-L\right) \\
& =\int d \sigma\left(N_{0} \mathcal{H}_{0}+N_{1} \mathcal{H}_{1}+B_{0} \chi\right),
\end{aligned}
$$

where

$$
\begin{aligned}
\mathcal{H}_{0} & =-\frac{1}{2 \rho} \Pi_{\mu} \Pi^{\mu}-\frac{1}{2} \rho \partial_{1} X_{\mu} \partial_{1} X^{\mu}+g_{11} E-\left(\partial_{1} \pi^{1}{ }_{a}+B_{1} \pi^{1}{ }_{b} \epsilon^{b}{ }_{a}\right) \epsilon^{a c} e_{1 c} \\
\mathcal{H}_{1} & =\Pi_{\mu} \partial_{1} X^{\mu}-\left(\partial_{1} \pi^{1}{ }_{a}+B_{1} \pi^{1}{ }_{b} \epsilon^{b}{ }_{a}\right) e_{1}{ }^{a} \\
\chi & =-\partial_{1} \pi^{1}+\pi^{1}{ }_{a} \epsilon^{a b} e_{1 b}
\end{aligned}
$$

and we have used the abbreviation

$$
E=\frac{1}{2 \beta} \pi^{1}{ }_{a} \pi^{1 a}-\frac{1}{4 \gamma} \pi^{1} \pi^{1}-\lambda .
$$

Equal time Poisson brakets between conjugate variables in points $\sigma$ and $\sigma^{\prime}$ have the usual form

$$
\begin{aligned}
\left\{X^{\mu}, \Pi_{\nu}^{\prime}\right\} & =\delta_{\nu}^{\mu} \delta\left(\sigma-\sigma^{\prime}\right), \\
\left\{N_{0}, P_{0}^{\prime}\right\} & =\delta\left(\sigma-\sigma^{\prime}\right), \\
\left\{N_{1}, P_{1}^{\prime}\right\} & =\delta\left(\sigma-\sigma^{\prime}\right), \\
\left\{e_{1}^{a}, \pi^{\prime 1}{ }_{b}\right\} & =\delta_{b}^{a} \delta\left(\sigma-\sigma^{\prime}\right), \\
\left\{B_{\alpha}, \pi^{\prime \beta}\right\} & =\delta_{\alpha}^{\beta} \delta\left(\sigma-\sigma^{\prime}\right) .
\end{aligned}
$$

Here primes denote the space point at which the variables are considered.

The functions $\mathcal{H}_{0}, \mathcal{H}_{1}$, and $\chi$ do not depend on $N_{0}, N_{1}$, and $B_{0}$. Therefore the secondary constraints are easily obtained considering equations of motion of primary constraints

$$
\begin{aligned}
& \partial_{0} P_{0}=\left\{P_{0}, H\right\}=-\mathcal{H}_{0} \approx 0, \\
& \partial_{0} P_{1}=\left\{P_{1}, H\right\}=-\mathcal{H}_{1} \approx 0, \\
& \partial_{0} \pi^{0}=\left\{\pi^{0}, H\right\}=-\chi \approx 0 .
\end{aligned}
$$

So the theory contains 3 secondary constraints, and the Hamiltonian (16) equals to their linear combination. Therefore evolution of the secondary constraints is entirely defined by the Poisson bracket algebra of the constraints. Straightforward calculations show that the secondary constraints satisfy the following algebra

$$
\begin{aligned}
\left\{\mathcal{H}_{0}, \mathcal{H}_{0}^{\prime}\right\} & =\left(\mathcal{H}_{1}+\mathcal{H}_{1}^{\prime}\right) \delta^{\prime}\left(\sigma^{\prime}-\sigma\right), \\
\left\{\mathcal{H}_{0}, \mathcal{H}_{1}^{\prime}\right\} & =\left(\mathcal{H}_{0}+\mathcal{H}_{0}^{\prime}\right) \delta^{\prime}\left(\sigma^{\prime}-\sigma\right)+\frac{1}{4} g_{11} \pi^{1} \chi \delta\left(\sigma^{\prime}-\sigma\right), \\
\left\{\mathcal{H}_{1}, \mathcal{H}_{1}^{\prime}\right\} & =\left(\mathcal{H}_{1}+\mathcal{H}_{1}^{\prime}\right) \delta^{\prime}\left(\sigma^{\prime}-\sigma\right), \\
\left\{\chi, \mathcal{H}_{0}^{\prime}\right\} & =\left\{\chi, \mathcal{H}_{1}^{\prime}\right\}=\left\{\chi, \chi^{\prime}\right\}=0,
\end{aligned}
$$

where primes on constraints denote their argument $\sigma^{\prime}$, while $\delta^{\prime}$ denotes the derivative of the $\delta$-function,

$$
\delta^{\prime}\left(\sigma^{\prime}-\sigma\right)=\partial_{\sigma} \delta\left(\sigma^{\prime}-\sigma\right) .
$$


It is clear also that the primary constraints commute between themselves and with the secondary constraints.

So the primary and secondary constraints are of the first class in Dirac's terminology. The Hamiltonial system is in involution, and there is no need to compute tertiary constraints.

The obtained Hamiltonian equals to the linear combination of the first class constraints. This is a common feature of the theories invariant under general coordinate transformation. In contrast to general relativity all constraints are polinomial in generalized coordinates and momenta. This is a particular feature of two dimensions.

Let us briefly discuss the geometrical meaning of the constraints. The integral

$$
Q_{L}=-\int d \sigma \omega \chi
$$

where $\omega$ is a parameter of local Lorentz rotation, generates correct Lorentz rotation (6) for the fields $e_{1}^{a}, B_{1}$, and $X^{\mu}$. For example,

$$
\delta B_{1}=\left\{B_{1}, Q_{L}\right\}=\partial_{1} \omega
$$

In the same way one may check that the integral

$$
Q_{1}=-\int d \sigma \epsilon^{1} \mathcal{H}_{1}
$$

generates coordinate transformations (5) for the same fields with the parameter $\delta \zeta^{\alpha}=\epsilon^{1} \delta_{1}^{\alpha}$. The integral

$$
Q_{0}=-\int d \sigma \epsilon^{0} \mathcal{H}_{0}
$$

is necessary to generate correct transformation of the time coordinate $\delta \zeta^{\alpha}=\epsilon^{0} \delta_{0}^{\alpha}$. In the latter case one must exclude time derivatives from (5) using the equations of motion, which in their turn are defined by the Hamiltonian. We do not discuss this subtle point here and only note that in the conformal gauge $N_{0}=1, N_{1}=B_{1}=0$ the constraint $\mathcal{H}_{0}$ represents the energy density.

\subsection{The algebra of the constraints}

The Poisson bracket algebra of the constraints (22) is not a true algebra because there are structure functions instead of structure constants. Let us pose the question whether there exists another equivalent set of constraints forming a true algebra? This question was solved in [13 by considering more general algebra including the momenta besides the constraints. The algebra was shown to be that of quadratically deformed iso(2,1)algebra. However there does exist the equivalent set of constraints by themselves satisfying the closed algebra. We show that this algebra is a semidirect sum of the Virasoro algebra and the one dimensional abelian algebra corresponding to the Lorentz rotation.

Let us define the new set of constraints

$$
\begin{aligned}
& \widetilde{\mathcal{H}}_{0}=\mathcal{H}_{0}+B_{1} \chi, \\
& \widetilde{\mathcal{H}}_{1}=\mathcal{H}_{1}+B_{1} \chi,
\end{aligned}
$$


the constraint $\chi$ being unchanged. Then straightforward calculations show that the new algebra closes with the structure constants instead of structure functions

$$
\begin{aligned}
\left\{\widetilde{\mathcal{H}}_{0}, \widetilde{\mathcal{H}_{0}^{\prime}}\right\} & =\left(\widetilde{\mathcal{H}_{1}}+\widetilde{\mathcal{H}}_{1}^{\prime}\right) \delta^{\prime}\left(\sigma^{\prime}-\sigma\right), \\
\left\{\widetilde{\mathcal{H}}_{0}, \widetilde{\mathcal{H}}_{1}^{\prime}\right\} & =\left(\widetilde{\mathcal{H}}_{0}+\widetilde{\mathcal{H}}_{0}^{\prime}\right) \delta^{\prime}\left(\sigma^{\prime}-\sigma\right), \\
\left\{\widetilde{\mathcal{H}}_{1}, \widetilde{\mathcal{H}}_{1}^{\prime}\right\} & =\left(\widetilde{\mathcal{H}_{1}}+\widetilde{\mathcal{H}_{1}^{\prime}}\right) \delta^{\prime}\left(\sigma^{\prime}-\sigma\right), \\
\left\{\chi, \widetilde{\mathcal{H}}_{0}^{\prime}\right\} & =\chi^{\prime} \delta^{\prime}\left(\sigma^{\prime}-\sigma\right), \\
\left\{\chi, \widetilde{\mathcal{H}}_{1}^{\prime}\right\} & =\chi^{\prime} \delta^{\prime}\left(\sigma^{\prime}-\sigma\right), \\
\left\{\chi, \chi^{\prime}\right\} & =0
\end{aligned}
$$

The first three Poisson brackets show that $\widetilde{\mathcal{H}}_{0}$ and $\widetilde{\mathcal{H}}_{1}$ form the Virasoro or the conformal algebra. The abelian constraint $\chi$ form an invariant subalgebra under the action of the Virasoro generators. So the new algebra is a semidirect sum of the Virasoro algebra and the one-dimensional abelian algebra. This algebra corresponds to the semidirect product of the conformal symmetry transformations on the Lorentz rotation.

Let us rewrite the algebra (24) in other forms to compare it with the Fourier transformed Virasoro algebra. Introducing generators

$$
\mathcal{H}^{ \pm}=\frac{1}{2}\left(\widetilde{\mathcal{H}}_{0} \pm \widetilde{\mathcal{H}}_{1}\right)
$$

the algebra takes the form

$$
\begin{aligned}
\left\{\mathcal{H}^{+}, \mathcal{H}^{\prime+}\right\} & =\left(\mathcal{H}^{+}+\mathcal{H}^{\prime+}\right) \delta^{\prime}\left(\sigma^{\prime}-\sigma\right), \\
\left\{\mathcal{H}^{+}, \mathcal{H}^{\prime-}\right\} & =0, \\
\left\{\mathcal{H}^{-}, \mathcal{H}^{\prime-}\right\} & =-\left(\mathcal{H}^{-}+\mathcal{H}^{\prime-}\right) \delta^{\prime}\left(\sigma^{\prime}-\sigma\right), \\
\left\{\chi, \mathcal{H}^{\prime+}\right\} & =\chi^{\prime} \delta^{\prime}\left(\sigma^{\prime}-\sigma\right), \\
\left\{\chi, \mathcal{H}^{\prime-}\right\} & =\left\{\chi, \chi^{\prime}\right\}=0 .
\end{aligned}
$$

We see that the algebra generated by $\mathcal{H}^{-}$completely decouples, and we are left with the semidirect sum of the algebras generated by $\mathcal{H}^{+}$and $\chi$.

The asymmetry between $\mathcal{H}^{+}$and $\mathcal{H}^{-}$is insignificant. If one redifines the generator $\widetilde{\mathcal{H}}_{0}$ in (23) using the expression $\widetilde{\mathcal{H}}_{0}=\mathcal{H}_{0}-B_{1} \chi$ and leaves the definition of $\widetilde{\mathcal{H}}_{1}$ and $\mathcal{H}^{ \pm}$unchanged, then decoupled is the algebra generated by $\mathcal{H}^{+}$. This shows that the symmetry between $\mathcal{H}^{+}$and $\mathcal{H}^{-}$in (25) was broken by the definition (23).

Let us make the Fourier transform

$$
\begin{aligned}
L_{m}^{ \pm} & =\int_{-\pi}^{\pi} d \sigma \mathcal{H}^{ \pm} e^{-\imath m \sigma}, \\
M_{m} & =\int_{-\pi}^{\pi} d \sigma \chi e^{-\imath m \sigma} .
\end{aligned}
$$

Then the algebra looks as follows

$$
\begin{aligned}
\left\{L_{m}^{+}, L_{n}^{+}\right\} & =\imath(m-n) L_{m+n}^{+}, \\
\left\{L_{m}^{-}, L_{n}^{-}\right\} & =-\imath(m-n) L_{m+n}^{-}, \\
\left\{L_{m}^{+}, M_{n}\right\} & =\imath m M_{m+n}, \\
\left\{L_{m}^{+}, L_{n}^{-}\right\} & =\left\{L_{m}^{-}, M_{n}\right\}=\left\{M_{m}, M_{n}\right\}=0 .
\end{aligned}
$$


Thus we have analysed the algebraic structure of the constraints of the string with dynamical geometry. The theory contains 6 first class constraints. Three primary first class constraints are abelian and completely decouple from the algebra, while 3 secondary first class constraints form the nontrivial generalization of the Virasoro algebra.

\subsection{Extra constraint in two-dimensional gravity with torsion}

In the case of pure two-dimensional gravity with torsion there is one extra first class constraint. In this section we assume that the string coordinates are absent.

Direct integration of the equations of motion of two-dimensional gravity with torsion [6, [] shows that there is the invariant relation between scalar curvature and torsion

$$
\left(\frac{\gamma^{2}}{\beta^{2}} R^{2}+2 \frac{\gamma}{\beta} R+2+\frac{4 \lambda \gamma}{\beta^{2}}+\frac{\gamma}{\beta} T_{a b c} T^{a b c}\right) e^{-\gamma R / \beta}=A,
$$

where $A$ is an arbitrary constant (integral of motion) defined by the initial data. This relation holds for any solution of the equations of motion. Comparing (27) with the definition of the momenta (14) and (15) we see that the integral of motion can be expressed intirely in terms of the canonical momenta

$$
A=-\frac{4 \gamma}{\beta^{2}}\left(E+\frac{\beta}{2 \gamma} \pi^{1}-\frac{\beta^{2}}{2 \gamma}\right) e^{\pi^{1} / \beta}
$$

where $E$ is defined by (20). Because $\mathrm{A}$ is the integral of motion and the Hamiltonian equals to the linear combination of the first class constraints, the expression (28) must be a first class constraint. Indeed, straightforward calculations yield the following Poisson brackets

$$
\begin{aligned}
\left\{A, \mathcal{H}^{\prime+}\right\} & =\frac{4 \gamma}{\beta^{3} g_{11}}\left(\pi^{1 a} e_{1 a}-\pi^{1 a} \epsilon_{a b} e_{1}^{b}\right) e^{\pi^{1} / \beta} \mathcal{H}^{+} \delta\left(\sigma^{\prime}-\sigma\right) \\
& +\frac{4 \gamma}{\beta^{3} g_{11}}\left[g_{11} E-B_{1}\left(\pi^{1 a} e_{1 a}-\pi^{1 a} \epsilon_{a b} e_{1}^{b}\right)\right] e^{\pi^{1} / \beta} \chi \delta\left(\sigma^{\prime}-\sigma\right) \\
\left\{A, \mathcal{H}^{\prime-}\right\} & =\frac{4 \gamma}{\beta^{3} g_{11}}\left(\pi^{1 a} e_{1 a}+\pi^{1 a} \epsilon_{a b} e_{1}^{b}\right) e^{\pi^{1} / \beta} \mathcal{H}^{-} \delta\left(\sigma^{\prime}-\sigma\right) \\
\left\{A, \chi^{\prime}\right\} & =\left\{A, A^{\prime}\right\}=0 .
\end{aligned}
$$

At first sight $A$ is an extra independent first class constraint of two-dimensional gravity with torsion. But this is not so. One may check that its space derivative is the linear combination of the secondary constraints for $X^{\mu}=0$

$$
\partial_{1} A=\frac{4 \gamma}{\beta^{3}}\left(\frac{e_{1}^{a} \epsilon_{a b} \pi^{1 b}}{g_{11}} \mathcal{H}_{0}+\frac{e_{1 a} \pi^{1 a}}{g_{11}} \mathcal{H}_{1}+E \chi\right) e^{\pi^{1 / \beta}}
$$

So it does not generate new gauge symmetry [14]. In fact, explicit integrability of the right hand side of (29) is connected to the integrability of pure two dimensional gravity with torsion. 


\subsection{The second canonical transformation}

Having in hand the Hamiltonian theory one can try to quantize it. By this we mean the explicit construction of the Fock space where all the operators will work. In Ref. [18] two-dimensional gravity with torsion is quantized using other representation of the operators with a symmetric ordering of the coordinates and momenta without introduction of creation and annihilation operators. Hopefully, different schemes of quantization will add new features to the theory.

To construct the Fock space one needs to introduce creation and annihilation operators. This can be done in the full nonlinear theory through the coordinates and momenta in the usual way without referring to the equations of motion. Then all the operators including the constraints must be supplemented by the normal ordering prescription. At this point one faces the problem of quantum anomalies because the algebra of the constraints must be closed also at the quantum level. Remember that in the theory of ordinary bosonic theory the anomaly exists. It is the central charge of the Virasoro algebra which is compensated by the ghost contribution in $D=26$.

In the case of the string with dynamical geometry the constraints are polinomials of the fourth order in creation and annihilation operators, and the calculation of the anomalies in the algebra can be, in principle, performed. But I failed to do this. Either the calculations become unmanagable or the central charge diverge. This problem forces me to make three more canonical transformations before the introduction of the creation and annihilation operators. The first was already done when we chose the canonical coordinates in the Lagrangian and here we consider the second most important canonical transformation.

To simplify the form of the constraints let us make the second nonlocal canonical transformation

$$
e_{1}^{a}, \pi_{a}^{1}, B_{1}, \pi^{1} \rightarrow Q, Q_{\perp}, P, P_{\perp}, Q_{B}, P_{B}
$$

corresponding to the separation of the longitudinal and transversal part of the zweibein. The string coordinates and momenta remain unchanged. May be this very peculiar canonical transformation is the most significant technical achievement of the present paper. We choose the generating function depending on old coordinates and new momenta

$$
F_{2}=P \frac{e_{1}^{a} \partial_{1} e_{1 a}}{g_{11}}+P_{\perp} \frac{e_{1}^{a} \epsilon_{a b} \partial_{1} e_{1}^{b}}{g_{11}}-P_{\perp} B_{1}+P_{B} B_{1}
$$

Then one can find the expressions for old momenta and new coordinates

$$
\begin{aligned}
\pi_{a}^{1} & =\frac{\delta F_{2}}{\delta e_{1}{ }^{a}}=-\partial_{1} P \frac{e_{1 a}}{g_{11}}-\partial_{1} P_{\perp} \frac{e_{1}^{b} \epsilon_{b a}}{g_{11}} \\
\pi^{1} & =\frac{\delta F_{2}}{\delta B_{1}}=-P_{\perp}+P_{B} \\
Q & =\frac{\delta F_{2}}{\delta P}=\frac{e_{1}{ }^{a} \partial_{1} e_{1 a}}{g_{11}} \\
Q_{\perp} & =\frac{\delta F_{2}}{\delta P_{\perp}}=\frac{e_{1}^{a} \epsilon_{a b} \partial_{1} e_{1}^{b}}{g_{11}}-B_{1} \\
Q_{B} & =\frac{\delta F_{2}}{\delta P_{B}}=B_{1} .
\end{aligned}
$$

The first of the above equations show that $\partial_{1} P$ and $\partial_{1} P_{\perp}$ represent the longitudinal and transversal part of $\pi^{1}{ }_{a}$ correspondingly. The appearence of the partial derivative $\partial_{1}$ in 
(31) means that the canonical transformation is nonlocal. It will be shown however that the nonlocality is inessential when the theory is considered on a cylinder corresponding to a closed string.

Calculating the right hand side of (31) we use the identity

$$
\frac{\delta}{\delta e_{1}^{a}} \int d \sigma \frac{e_{1}^{a} \epsilon_{a b} \partial_{1} e_{1}^{b}}{g_{11}}=0
$$

This piculiar identity is proved by direct calculation and is nontrivial because the integrand is not equal to a total divergence.

The second used identity

$$
\frac{\delta}{\delta e_{1}^{a}} \int d \sigma \frac{e_{1}^{a} \partial_{1} e_{1 a}}{g_{11}}=0
$$

is trivial because the integrand is a total divergence

$$
\frac{e_{1}^{a} \partial_{1} e_{1 a}}{g_{11}}=\frac{1}{2} \partial_{1} \ln \left(-g_{11}\right), \quad g_{11}<0 .
$$

Using Eqs.(31) the constraints can be written in the form

$$
\begin{aligned}
\mathcal{H}_{0} & =-\frac{1}{2 \rho} \Pi_{\mu} \Pi^{\mu}-\frac{1}{2} \rho \partial_{1} X_{\mu} \partial_{1} X^{\mu} \\
& +\frac{1}{2 \beta}\left(\partial_{1} P\right)^{2}-\frac{1}{2 \beta}\left(\partial_{1} P_{\perp}\right)^{2}-\partial_{1} P Q_{\perp}-\partial_{1} P_{\perp} Q+\partial_{1}^{2} P_{\perp}-\frac{1}{4 \gamma} g_{11}\left(P_{\perp}-P_{B}\right)^{2}-\lambda g_{11}, \\
\mathcal{H}_{1} & =\Pi_{\mu} \partial_{1} X^{\mu}-\partial_{1} P Q-\partial_{1} P_{\perp} Q_{\perp}+\partial_{1}^{2} P, \\
\chi & =-\partial_{1} P_{B} .
\end{aligned}
$$

The constraint $\mathcal{H}_{0}$ is quadratic in the fields except the linear term and two last terms containing nonpolinomial space component of the metric

$$
g_{11}=-\exp \left(2 \int_{\sigma_{0}}^{\sigma} d \sigma^{\prime} Q\left(\tau, \sigma^{\prime}\right)\right) .
$$

This is the consequence of the nonlocality of the canonical transformation. The constant of integration $\sigma_{0}$ is just the point where $g_{11}=-1$. So at this point the unit of space length is chosen to be unity at every moment of time. The second constraint $\mathcal{H}_{1}$ contains quadratic and one linear terms. The last constraint $\chi$ is linear.

To clarify the geometric meaning of the second canonical transformation let us parametrize the zweibein components $e_{1}^{a}$ by the Lorentz angle $\omega$ and the conformal factor $e^{\varphi}>$ 0 , where $\varphi(\xi)$ is a "scalar" field. Indeed, $e_{1}{ }^{a}$ is a Lorentz vector and can be always parametrized as follows

$$
e_{1}^{a}=e^{\varphi}\left(n^{a} \cosh \omega+\epsilon^{a b} n_{b} \sinh \omega\right), \quad g_{11}=-e^{2 \varphi} .
$$

where $n^{a}$ is a fixed spacelike vector of unit length $n^{a} n_{a}=-1$. Then Eqs.(31) imply

$$
Q=\partial_{1} \varphi, \quad Q_{\perp}=\partial_{1} \omega
$$

So longitudinal and transversal components $Q, Q_{\perp}$ are parametrized by the comformal factor and the Lorentz angle. The important point is that the initial theory is invariant 
under local Lorentz rotation. This sugests that the modes corresponding to $Q_{\perp}, P_{\perp}$ are unphysical and can be always gauged away.

The second canonical transformation is not a one-to-one transformation. The first equation (31) shows that $P$ and $P_{\perp}$ are defined through the original coordinates and momenta up to an addition of arbitrary constants. So dealing with new canonical variables one can add arbitrary constants to $P$ and $P_{\perp}$ and the corresponding constant to $P_{B}$ without changing the initial theory. This freedom will be used in the following section. At the same time the new set of canonical variables also does not uniquely define the original coordinates. The conformal factor $\varphi$ and the Lorentz angle $\omega$ are defined by (35) up to an arbitrary constants too.

\subsection{Fixing of the gauge}

According to a general prescription one can introduce creation and annihilation operators for all fields describing both physical and unphysical degrees of freedom. Then one must check whether the algebra of the constraints is satisfied at the quantum level too.

String with dynamical geometry is described by $D+6$ pairs of canonically conjugate variables. Six first class constraints together with six gauge conditions can be used to eliminate 6 unphysical degrees of freedom. So the string with dynamical geometry contains $D$ physical degrees of freedom.

To quantize the model we use the gauge freedom only partially. Namely, we eliminate 4 unphysical degrees of freedom explicitly and leave 2 unphysical degrees of freedom and 2 first class constraints satisfying the pure Virasoro algebra. This has been done to simplify the quantization procedure and to be as close to the ordinary bosonic string theory as possible.

The extended Hamiltonian of the string with dynamical geometry equals to the linear combination of all constraints

$$
H_{E}=\int d \sigma\left(v_{0} P_{0}+v_{1} P_{1}+v \pi^{0}+u_{0} \mathcal{H}_{0}+u_{1} \mathcal{H}_{1}+u \chi\right)
$$

where $v_{0}, v_{1}, v, u_{0}, u_{1}, u$ are Lagrange multiplyers. According to a general prescription one can choose one gauge condition for every first class constraint. Every gauge condition together with the corresponding first class constraint must form a pair of second class constraints.

Consider 3 primary first class constraints (12). We choose the conformal gauge for the metric

$$
N_{0}=1, \quad N_{1}=0,
$$

and fix the time component of the Lorentz connection

$$
B_{0}=\text { const. }
$$

Gauge conditions (37) and (38) clearly make a pairs of second class constraints with the primary constraints. They must be also preserved in time. The corresponding equations of motion are

$$
\begin{aligned}
& \partial_{0} N_{0}=\left\{N_{0}, H_{E}\right\}=v_{0}, \\
& \partial_{0} N_{1}=\left\{N_{1}, H_{E}\right\}=v_{1}, \\
& \partial_{0} B_{0}=\left\{B_{0}, H_{E}\right\}=v .
\end{aligned}
$$


So the gauge choice (37) and (38) fixes 3 Lagrange multipliers in the extended Hamiltonian

$$
v_{0}=v_{1}=v=0
$$

Now we consider the remaining degree of freedom of the Lorentz connection described by $Q_{B}$ and $P_{B}$. Equations of motion for these variables are

$$
\begin{aligned}
\partial_{0} Q_{B} & =\frac{1}{2} u_{0} g_{11}\left(P_{B}-P_{\perp}\right)+\partial_{1} u \\
\partial_{0} P_{B} & =-\left(u_{0}+u_{1}\right) \chi
\end{aligned}
$$

We choose the following gauge condition

$$
Q_{B}=\text { const }
$$

It forms a pair of second class constraints with the secondary constraints $\mathcal{H}_{0}$ and $\chi$. The constraint $\chi=0$ together with the equation of motion (40) yields

$$
P_{B}=c_{B}=\text { const, }
$$

where $c_{B}$ is an arbitrary constant. As was noted in the preceeding section addition of arbitrary constant to $P_{B}$ and the corresponding constant to $P_{\perp}$ does not change the initial theory. So without loss of generality we set $c_{B}=0$.

The gauge condition (41) must be consistent with the equation of motion (39). This defines the Lagrange multiplier $u$ in terms of $u_{0}$

$$
\frac{1}{2} u_{0} g_{11}\left(P_{B}-P_{\perp}\right)+\partial_{1} u=0
$$

The corresponding Hamiltonian reads

$$
\widehat{H}_{E}=\int d \sigma\left(u^{0} \widehat{\mathcal{H}}_{0}+u^{1} \mathcal{H}_{1}\right),
$$

where the hatted constraint $\widehat{\mathcal{H}}_{0}$ is obtained from the initial constraint $\mathcal{H}_{0}$ by setting $P_{B}=0$

$$
\begin{aligned}
\widehat{\mathcal{H}}_{0} & =-\frac{1}{2 \rho} \Pi_{\mu} \Pi^{\mu}-\frac{\rho}{2} \partial_{1} X_{\mu} \partial_{1} X^{\mu} \\
& +\frac{1}{2 \beta}\left(\partial_{1} P\right)^{2}-\frac{1}{2 \beta}\left(\partial_{1} P_{\perp}\right)^{2}-\partial_{1} P Q_{\perp}-\partial_{1} P_{\perp} Q+\partial_{1}^{2} P_{\perp}-g_{11}\left(\frac{1}{4 \gamma} P_{\perp}^{2}+\lambda\right) .
\end{aligned}
$$

The constraints $\widehat{\mathcal{H}}_{0}$ and $\mathcal{H}_{1}$ as well as $\mathcal{H}_{0}$ and $\mathcal{H}_{1}$ form precisely the Virasoro algebra

$$
\begin{aligned}
& \left\{\widehat{\mathcal{H}}_{0}, \widehat{\mathcal{H}}_{0}^{\prime}\right\}=\left(\mathcal{H}_{1}+\mathcal{H}_{1}^{\prime}\right) \delta^{\prime}\left(\sigma^{\prime}-\sigma\right), \\
& \left\{\widehat{\mathcal{H}}_{0}, \mathcal{H}_{1}^{\prime}\right\}=\left(\widehat{\mathcal{H}}_{0}+\widehat{\mathcal{H}}_{0}^{\prime}\right) \delta^{\prime}\left(\sigma^{\prime}-\sigma\right), \\
& \left\{\mathcal{H}_{1}, \mathcal{H}_{1}^{\prime}\right\}=\left(\mathcal{H}_{1}+\mathcal{H}_{1}^{\prime}\right) \delta^{\prime}\left(\sigma^{\prime}-\sigma\right) .
\end{aligned}
$$

In this way we eliminate 4 unphysical degrees of freedom using 4 gauge conditions and 4 constraints. Four of the Lagrange multipliers are also fixed by the requirement of the consistency of the gauge conditions with the equations of motion. The net result is that we are left with $D+2$ degrees of freedom $\left(X^{\mu}, \Pi_{\mu}\right),(Q, P)$, and $\left(Q_{\perp}, P_{\perp}\right)$ subjected to two first class constraints satisfying the Virasoro algebra. 


\subsection{The third canonical transformation and positive definiteness of the Hamiltonian}

Although we considerably simplify the form of the constraints and reduce their number from 6 to 2 we are still unable to provide quantization. Therefore we will make the third canonical transformation

$$
Q, P, Q_{\perp}, P_{\perp} \rightarrow q, p, q_{\perp}, p_{\perp}
$$

which has the peculiar feature that the quadratic part of the resulting constraints precisely coinsides with the constraints of an ordinary bosonic string.

Let us choose the generating function

$$
F_{3}=-\partial_{1} P q-\partial_{1} P_{\perp} q_{\perp}+\beta q_{\perp} \partial_{1} q
$$

depending on the old momenta and new coordinates. It is also nonlocal and connects old and new variables

$$
\begin{aligned}
Q & =-\frac{\delta F_{3}}{\delta P}=-\partial_{1} q \\
Q_{\perp} & =-\frac{\delta F_{3}}{\delta P_{\perp}}=-\partial_{1} q_{\perp}, \\
p & =-\frac{\delta F_{3}}{\delta q}=\partial_{1} P+\beta \partial_{1} q_{\perp}, \\
p_{\perp} & =-\frac{\delta F_{3}}{\delta q_{\perp}}=\partial_{1} P_{\perp}-\beta \partial_{1} q .
\end{aligned}
$$

Then the factor $g_{11}$ in the constraints again becomes local

$$
g_{11}=-e^{2 \int Q}=-e^{-2 q},
$$

the constant of integration being inessential because it can be always normalized by choosing the appropriate value of $\sigma_{0}$ in (33). At the same time there arises other nonlocal quantity in the constraint $\widehat{\mathcal{H}}_{0}$ expressed through the new variables

$$
P_{\perp}=\int^{\sigma} d \sigma^{\prime}\left(p_{\perp}^{\prime}+\beta \partial_{1}^{\prime} q^{\prime}\right)=\int_{\sigma_{2}}^{\sigma} d \sigma^{\prime} p_{\perp}\left(\sigma^{\prime}\right)+\beta q,
$$

with some integration constant $\sigma_{2}$.

The third canonical transformation by itself is not a one-to-one transformation too. However, the combination of the second and the third canonical transformation yields a one-to-one transformation for zweibein $e_{1}{ }^{a}, \pi^{1}{ }_{a} \rightarrow q, q_{\perp}, p, p_{\perp}$ up to unessential addition of arbitrary constants to $q$ and $q_{\perp}$.

Comparing Eqs.(45) and (35) one finds the geometric meaning of the new variables. Coordinates $q$ and $q_{\perp}$ up to a constant coinside with the conformal factor $\varphi$ and the angle $\omega$ of $e_{1}^{a}$ in (34).

In terms of the new canonical variables the constraints read

$$
\begin{aligned}
\widehat{\mathcal{H}}_{0} & =-\frac{1}{2 \rho} \Pi_{\mu} \Pi^{\mu}-\frac{\rho}{2} \partial_{1} X_{\mu} \partial_{1} X^{\mu}+\frac{1}{2 \beta} p^{2}+\frac{\beta}{2}\left(\partial_{1} q\right)^{2}-\frac{1}{2 \beta} p_{\perp}^{2}-\frac{\beta}{2}\left(\partial_{1} q_{\perp}\right)^{2} \\
& +\partial_{1} p_{\perp}+\beta \partial_{1}^{2} q+e^{-2 q}\left(\frac{1}{4 \gamma} P_{\perp}^{2}+\lambda\right) \\
\mathcal{H}_{1} & =\Pi_{\mu} \partial_{1} X^{\mu}+p \partial_{1} q+p_{\perp} \partial_{1} q_{\perp}+\partial_{1} p-\beta \partial_{1}^{2} q_{\perp} .
\end{aligned}
$$


This form of the constraints deserves some discussion. It is quite suprising that the quadratic parts of the constraints (47), (48) precisely coinside with the string part of the constraints. Besides there are also linear terms and the exponential term. The last is nonlocal because of the presence of $P_{\perp}$ defined by (46). It is worth mensioning that constraints (47) and (48) look very similar to the constraints obtained in 115 where the string with dynamical geometry was canonically formulated after fixing the conformal guage in the equations of motion. It is not surprising because the coordinate $q$ coinsides with the Liouville mode. I fact one can show that constraints (47) and (48) are connected with the constraints in Ref. [15] by canonical transformation.

Let us set for a moment $q_{\perp}=p_{\perp}=0$ naively excluding the unphysical mode from the theory. Then the constraints read

$$
\begin{aligned}
\widehat{\mathcal{H}}_{0} & =-\frac{1}{2 \rho} \Pi_{\mu} \Pi^{\mu}-\frac{\rho}{2} \partial_{1} X_{\mu} \partial_{1} X^{\mu} \\
& +\frac{1}{2 \beta} p^{2}+\frac{\beta}{2}\left(\partial_{1} q\right)^{2}+\beta \partial_{1}^{2} q+\lambda e^{-2 q}+\frac{\beta^{2}}{4 \gamma} q^{2} e^{-2 q} \\
\mathcal{H}_{1} & =\Pi_{\mu} \partial_{1} X^{\mu}+p \partial_{1} q+\partial_{1} p .
\end{aligned}
$$

This form of the constraints up to the last term in (49) coinsides with the constraints in the string theory coupled to the Liouville field [24, 25, 26, 27]. Even the linear "improvement" term which was originally added to the Liouville part of the constraints by hand appears here automatically. At the same time the string with dynamical geometry is more general. The constraints (47) and (48) which quantum algebra will be elucidated in the second part of the paper depend from two fields $q$ and $q_{\perp}$ instead of one Liouville mode. This yields more freedom in the construction of the Fock space representation and there does exist such representation where exponential operators commute.

It is worth mentioning that the form of the constraints (49), (50) closely reminds the $\sigma$-model approach to the bosonic string. Indeed, one can consider $q$ and $q_{\perp}$ as two extra components of the string. The corresponding $D+2$ dimensional string moves in a curved embedding manifold with two timelike coordinates. The difference is that instead of $p_{\perp}$ one has its primitive in the exponent term.

We note that in four dimensions algebra of the constraints in general relativity is quite similar to the Virasoro algebra and one readily finds their stringy quadratic representation which is similar to the constraints in the membrane model. I suppose that there also exists the canonical transformation when the quadratic part of the constraints will have precisely the form of the string constraints. May be this approach will yeild consistent Fock space representation of the constraints in quantum gravity.

New form of the constraint (47) allows one to make an important conclusion. Namely, one of the most crucial point in quantum field theory is positive definiteness of the canonical Hamiltonian for physical modes. In the case of the string with dynamical geometry it is given by $\widehat{\mathcal{H}}_{0}$. We readily see that string space coordinates $X^{i}, i=1, \ldots, D-1$, yield positive contributions if and only if $\rho>0$. As noted in Sect.2.6 the variables $q_{\perp}, p_{\perp}$ can be always gauged away and are unphysical. We see that the rest of the Hamiltonian is positive definite if $\beta, \gamma>0, \lambda \geq 0$. To prove this one has only to absorb the linear term into the quadratic one, for example, in the momentum representation. Thus we showed that for

$$
\rho, \beta, \gamma>0, \quad \lambda \geq 0,
$$


the canonical Hamiltonian is positive definite for all physical modes. This statement is quite untrivial if one deals with the Hamiltonian $\mathcal{H}_{0}$ in the initial representation (17).

At the end of the first part of this paper we will make the final fourth one-to-one canonical transformation

$$
q, q_{\perp}, p, p_{\perp} \rightarrow q^{ \pm}, p^{ \pm}
$$

It is linear and defined by the generating function

$$
F_{4}=-\frac{1}{\sqrt{2}}\left(q^{+}+q^{-}\right) p-\frac{1}{\sqrt{2}}\left(q^{+}-q^{-}\right) p_{\perp}
$$

depending on the old momenta and new coordinates. Corresponding transformation formulas read

$$
\begin{aligned}
q^{+} & =\frac{1}{\sqrt{2}}\left(q+q_{\perp}\right), & p^{+} & =\frac{1}{\sqrt{2}}\left(p+p_{\perp}\right), \\
q^{-} & =\frac{1}{\sqrt{2}}\left(q-q_{\perp}\right), & p^{-} & =\frac{1}{\sqrt{2}}\left(p-p_{\perp}\right) .
\end{aligned}
$$

The new variables precisely coinside with the familiar light cone variables in the string theory. One can readily write down the constraints in terms of $q^{ \pm}$and $p^{ \pm}$. They will be considered in the next part of the paper. In this way we obtained the Hamiltonian formulation of the string with dynamical geometry suitable for quantization. I hope the reader is convinced that it is not so trivial.

\section{Canonical quantization}

Canonical quantization means that instead of the generalized coordinates and the conjugate momenta we must introduce noncomuting operators acting in some representation space. As usual for quantum field theory, we will construct the Fock space using the creation and annihilation operators and assume the normal ordering prescription for those operators which are nonlinear in coordinates and momenta. Next we compute explicitly the algebra of the quantum constraints and prove that two-dimensional gravity with torsion by itself is anomaly free, that is there are no extra terms as compared to the classical Poisson bracket algebra. So the central charge in the Virasoro algebra arise only from the string coordinates. Let us note that the ghost contribution does not taken into account in the present paper.

To the end of this paper we consider a closed string, that is all the fields are assumed to be periodic in space coordinate $\sigma$ with the period $2 \pi$. For definiteness we consider the interval $-\pi<\sigma<\pi$.

The scheme of quantization is usual. We expand all the fields in Fourier series, introduce creation and annihilation operators, construct the Fock space, find the normal ordered expressions for the Virasoro operators, and finally compute the quantum Virasoro algebra.

\subsection{The Fock space}

Here we introduce the creation and annihilation operators and construct the Fock space for the string with dynamical geometry. To avoid the problems arising from the space de- 
pendence of the basic operators in the theory we define creation and annihilation operators for the Fourier transforms of the fields.

The Fourier transform is defined as follows

$$
\begin{array}{rlrl}
X^{\mu} & =\frac{1}{\sqrt{2 \pi}} \sum_{k=-\infty}^{\infty} X_{k}^{\mu} e^{\imath k \sigma}, & X_{k}^{\mu}=\frac{1}{\sqrt{2 \pi}} \int_{-\pi}^{\pi} d \sigma X^{\mu} e^{-\imath k \sigma}, \\
\Pi^{\mu}=\frac{1}{\sqrt{2 \pi}} \sum_{k=-\infty}^{\infty} \Pi_{k}^{\mu} e^{\imath k \sigma}, & \Pi_{k}^{\mu}=\frac{1}{\sqrt{2 \pi}} \int_{-\pi}^{\pi} d \sigma \Pi^{\mu} e^{-\imath k \sigma}, \\
q^{ \pm}=\frac{1}{\sqrt{2 \pi}} \sum_{k=-\infty}^{\infty} q_{k}^{ \pm} e^{\imath k \sigma}, & q_{k}^{ \pm}=\frac{1}{\sqrt{2 \pi}} \int_{-\pi}^{\pi} d \sigma q^{ \pm} e^{-\imath k \sigma}, \\
p^{ \pm}=\frac{1}{\sqrt{2 \pi}} \sum_{k=-\infty}^{\infty} p_{k}^{ \pm} e^{\imath k \sigma}, & p_{k}^{ \pm}=\frac{1}{\sqrt{2 \pi}} \int_{-\pi}^{\pi} d \sigma p^{ \pm} e^{-\imath k \sigma} .
\end{array}
$$

It is clear that $X_{-k}^{\mu}=\left(X_{k}^{\mu}\right)^{*}$ where the star denotes complex conjugate. Similar relations hold for all other components.

The Fourier components satisfy the following nonvanishing Poisson bracket relations

$$
\begin{aligned}
\left\{X_{k}^{\mu}, \Pi_{\nu l}\right\} & =\delta_{\nu}^{\mu} \delta_{k,-l}, \\
\left\{q_{k}^{+}, p_{l}^{+}\right\} & =\left\{q_{k}^{-}, p_{l}^{-}\right\}=\delta_{k,-l} .
\end{aligned}
$$

To construct the quantum theory we replace the components of the fields by the operators which satisfy the canonical commutation relations obtained from the Poisson brackets by the replacement $\{\ldots\} \rightarrow-i[\ldots]$ where square brackets denote the commutator. Then the canonical commutation relations have the form

$$
\begin{aligned}
{\left[X_{k}^{\mu}, \Pi_{\nu l}\right] } & =\imath \delta_{\nu}^{\mu} \delta_{k,-l}, \\
{\left[q_{k}^{+}, p_{l}^{+}\right] } & =\left[q_{k}^{-}, p_{l}^{-}\right]=\imath \delta_{k,-l},
\end{aligned}
$$

all other commutators vanishing.

Let us introduce the annihilation and creation operators for nonzero modes $k \neq 0$

$$
\begin{aligned}
a_{k}^{\mu} & =\frac{1}{\sqrt{2}}\left(\sqrt{\rho|k|} X_{k}^{\mu}-\frac{i}{\sqrt{\rho|k|}} \Pi_{k}^{\mu}\right), \\
a_{k}^{* \mu} & =\frac{1}{\sqrt{2}}\left(\sqrt{\rho|k|} X_{-k}^{\mu}+\frac{i}{\sqrt{\rho|k|}} \Pi_{-k}^{\mu}\right) \\
a_{k} & =\frac{1}{\sqrt{2}}\left(\sqrt{\beta|k|} q_{k}^{+}+\frac{\imath}{\sqrt{\beta|k|}} p_{k}^{+}\right) \\
a_{k}^{*} & =\frac{1}{\sqrt{2}}\left(\sqrt{\beta|k|} q_{-k}^{+}-\frac{\imath}{\sqrt{\beta|k|}} p_{-k}^{+}\right) \\
b_{k} & =\frac{1}{\sqrt{2}}\left(\sqrt{\beta|k|} q_{k}^{-}-\frac{\imath}{\sqrt{\beta|k|}} p_{k}^{-}\right) \\
b_{k}^{*} & =\frac{1}{\sqrt{2}}\left(\sqrt{\beta|k|} q_{-k}^{-}+\frac{\imath}{\sqrt{\beta|k|}} p_{-k}^{-}\right) .
\end{aligned}
$$


Here and below the star denotes Hermitian conjugation of the operators.

Creation and annihilation operators for the string coordinates (54) and (55) are introduced in the usual way. This is done to show how the central charge arises and for comparison with the latter construction. In section 3.6 we show how to introduce another set of creation and annihilation operators producing no anomaly for $X^{0}$ and $X^{1}$ components of the string.

The zero modes of the string are treated in the Schrödinger coordinate representation. They act in the space of functions of $X_{0}^{\mu}$. That is the zero modes $X_{0}^{\mu}$ act as the ordinary multiplication on $X_{0}^{\mu}$ while the momenta act as the differention

$$
\Pi_{\mu 0}=-i \frac{\partial}{\partial X_{0}^{\mu}} .
$$

This representation of the zero modes of the string is justified by the physical interpretation. Indeed, the zero modes $X_{0}^{\mu}$ and $\Pi_{\mu 0}$ describe the position of the centre of mass and the total momentum of the string. Therefore their treating in the Schrödinger picture is similar to that of a point particle and describe the motion of the string as a whole in the embedding space. At the same time the creation operators $a_{k}^{* \mu}, k \neq 0$, describe the exited states corresponding to the oscillations of the string.

The zero modes $q_{0}^{ \pm}$and $p_{0}^{ \pm}$are treated in another way. We introduce for them the creation and annihilation operators as well as for nonzero modes

$$
\begin{aligned}
& a_{0}=\frac{1}{\sqrt{2}}\left(\sqrt{\beta} q_{0}^{+}+\frac{\imath}{\sqrt{\beta}} p_{0}^{+}\right), \\
& a_{0}^{*}=\frac{1}{\sqrt{2}}\left(\sqrt{\beta} q_{0}^{+}-\frac{\imath}{\sqrt{\beta}} p_{0}^{+}\right), \\
& b_{0}=\frac{1}{\sqrt{2}}\left(\sqrt{\beta} q_{0}^{-}-\frac{\imath}{\sqrt{\beta}} p_{0}^{-}\right), \\
& b_{0}^{*}=\frac{1}{\sqrt{2}}\left(\sqrt{\beta} q_{0}^{-}+\frac{\imath}{\sqrt{\beta}} p_{0}^{-}\right) .
\end{aligned}
$$

These relations are obtained from those for nonzero modes by setting $k=1$ in the right hand sides of (56)-(59).

This is the usual treatment of zero modes in quantum field theory. For example, in the scalar field theory or quantum electrodynamics the creation and annihilation operators are introduced for all modes including the zero ones. In our case the operators $a_{0}^{*}$ and $b_{0}^{*}$ create the exited states of constant metric while the operators $a_{k}^{*}$ and $b_{k}^{*}, k \neq 0$, create the states with the metric oscillating around some constant value.

At the beginning I treated the zero modes $q_{0}^{ \pm}$and $p_{0}^{ \pm}$in the Schrödinger picture like they are usually treated in the quantum Liouville theory. Both treatments yield the quantum theories free from anomalies. Therefore the treatment of zero modes concerns only the physical interpretation.

The formulas inverse to (54)-(63) expressing the Fourier components through the creation and annihilation operators look as follows

$$
X_{k}^{\mu}=\frac{1}{\sqrt{2 \rho|k|}}\left(a_{k}^{\mu}+a_{-k}^{* \mu}\right),
$$




$$
\begin{aligned}
\Pi_{k}^{\mu} & =\imath \sqrt{\frac{\rho|k|}{2}}\left(a_{k}^{\mu}-a_{-k}^{* \mu}\right), \\
q_{k}^{+} & =\frac{1}{\sqrt{2 \beta|k|}}\left(a_{k}+a_{-k}^{*}\right), \\
p_{k}^{+} & =-\imath \sqrt{\frac{\beta|k|}{2}}\left(a_{k}-a_{-k}^{*}\right), \\
q_{k}^{-} & =\frac{1}{\sqrt{2 \beta|k|}}\left(b_{k}+b_{-k}^{*}\right), \\
p_{k}^{-} & =\imath \sqrt{\frac{\beta|k|}{2}}\left(b_{k}-b_{-k}^{*}\right),
\end{aligned}
$$

for $k \neq 0$ and

$$
\begin{aligned}
q_{0}^{+} & =\frac{1}{\sqrt{2 \beta}}\left(a_{0}+a_{0}^{*}\right), \\
p_{0}^{+} & =-\imath \sqrt{\frac{\beta}{2}}\left(a_{0}-a_{0}^{*}\right), \\
q_{0}^{-} & =\frac{1}{\sqrt{2 \beta}}\left(b_{0}+b_{0}^{*}\right), \\
p_{0}^{-} & =\sqrt{\frac{\beta}{2}}\left(b_{0}-b_{0}^{*}\right),
\end{aligned}
$$

for zero modes.

Annihilation and creation operators satisfy the canonical commutation relations

$$
\begin{aligned}
{\left[a_{k}^{\mu}, a_{l}^{* \nu}\right] } & =-\eta^{\mu \nu} \delta_{k, l}, \\
{\left[a_{k}, a_{l}^{*}\right] } & =\delta_{k, l}, \\
{\left[b_{k}, b_{l}^{*}\right] } & =-\delta_{k, l},
\end{aligned}
$$

all other commutators vanishing. In formulas (75) and (76) the indices may take zero values.

Let us note the difference in the introduction of annihilation and creation operators. The operators $a_{k}$ and $a_{k}^{*}$ satisfy the same commutation relation as the operators $a_{k}^{i}$ and $a_{k}^{* i}, i=1, \ldots, D-1$, corresponding to the space string components. Operators $b_{k}$ and $b_{k}^{*}$ are similar to $a_{k}^{0}$ and $a_{k}^{* 0}$ corresponding to the time component of the string. Note that in the definitions (54), (55) the momenta enter with the upper Greek index whereas the momenta conjugate to $X^{\mu}$ (13) have lower index. So for the space components $\Pi^{i}=-\Pi_{i}$ the definition (54) has the same form as (56). This difference is obvious if one introduces annihilation and creation operators for $p_{\perp}$ and $p$ because $p_{\perp}$ yields negative contribution to the energy of the string and thus must produce indefinite metric in the Fock space. Going from $p_{\perp}, p$ to $p^{ \pm}$one can choose either the definition (56), (58) or similar definitions with $q^{+}, p^{+}$and $q^{-}, p^{-}$interchanged. In fact, the only essential point is that the commutators (75), (76) have opposite signs.

The vacuum $\mid 0>$ is defined as follows

$$
a_{k}^{\mu}\left|0>=a_{k}\right| 0>=b_{k} \mid 0>=0
$$


for all annihilation operators. By definition it does not depend on $X_{0}^{\mu}$

$$
\Pi_{\mu 0} \mid 0>=0 .
$$

The eigenstates with total momentum $k_{\mu}$ are defined as usual

$$
\begin{aligned}
\mid 0, k_{\mu}> & =e^{i k_{\mu} X_{0}^{\mu}} \mid 0>, \\
\Pi_{\mu 0} \mid 0, k_{\mu}> & =k_{\mu} \mid 0, k_{\mu}>.
\end{aligned}
$$

The exited states of the Fock space are defined by the action of a number of the creation operators on the vacuum with the corresponding normalization factors

$$
\left|N_{1}, N_{2}, \ldots, k_{\mu}>=\frac{1}{\sqrt{N_{1} !}}\left(A_{1}^{*}\right)^{N_{1}} \frac{1}{\sqrt{N_{2} !}}\left(A_{2}^{*}\right)^{N_{2}} \ldots\right| 0, k_{\mu}>,
$$

where $A_{K}^{*}, K=1,2, \ldots$ denotes one of the infinite set of the creation operators $\left\{A_{K}^{*}\right\}=$ $\left\{a_{k}^{* \mu}, a_{k}^{*}, b_{k}^{*}\right\}$. These vectors are normalised as follows

$$
<k_{\mu}^{\prime}, N_{1}^{\prime}, N_{2}^{\prime}, \ldots \mid N_{1}, N_{2}, \ldots, k_{\mu}>= \pm \delta_{N_{1}, N_{1}^{\prime}} \delta_{N_{2}, N_{2}^{\prime}} \ldots(2 \pi)^{D} \delta^{D}\left(k_{\mu}-k_{\mu}^{\prime}\right),
$$

where the integration over $X_{0}^{\mu}$ is understood.

So we have constructed the Fock space. It has indefinite metric because the action of an odd number of the creation operators $a_{k}^{* 0}$ and $b_{k}^{*}$ yields negative norm states. In the present paper we do not adress the important question of elimination of these states from the physical spectrum and concentrate our attention on the quantum algebra of the constraints.

The constraints are quadratic in $X^{\mu}, \Pi_{\mu}, p^{ \pm}$, and are nonpolinomial in $q^{ \pm}$because of the presence of the exponent term. To obtain from them the Hermitian operators acting in the Fock space we adopt the normal ordering prescription denoted by colons. That is all creation operators should be written on the left. In the next sections we compute the quantum algebra of the constraints.

\subsection{The quantum constraints}

This is a preparatory section containing the expressions of the constraints through creation and annihilation operators and the expressions for various contractions used in the following calculations.

To make the computations managable let us divide the constraints into 8 pieces

$$
\begin{aligned}
& \mathcal{H}^{+}=\mathcal{H}_{X}^{+}+\mathcal{H}_{(1)}^{+}+\mathcal{H}_{(a b)}+\mathcal{H}_{(a a)}+\mathcal{H}_{(b b)}+\mathcal{H}_{(e x p)}, \\
& \mathcal{H}^{-}=\mathcal{H}_{X}^{-}+\mathcal{H}_{(1)}^{-}+\mathcal{H}_{(a b)}-\mathcal{H}_{(a a)}-\mathcal{H}_{(b b)}+\mathcal{H}_{(e x p)} .
\end{aligned}
$$

Here $\mathcal{H}_{X}^{ \pm}$are the familiar quadratic constraints for a string coordinates

$$
\mathcal{H}_{X}^{ \pm}=-: \frac{1}{4}\left(\frac{1}{\sqrt{\rho}} \Pi_{\mu} \mp \sqrt{\rho} \partial_{1} X_{\mu}\right)\left(\frac{1}{\sqrt{\rho}} \Pi^{\mu} \mp \sqrt{\rho} \partial_{1} X^{\mu}\right):
$$

where colons denote the normal ordering. The terms $\mathcal{H}_{(1)}^{ \pm}$are linear in $q^{ \pm}$and $p^{ \pm}$

$$
\begin{aligned}
& \mathcal{H}_{(1)}^{+}=\frac{1}{\sqrt{2}} \partial_{1} p^{+}+\frac{1}{\sqrt{2}} \beta \partial_{1}^{2} q^{-} \\
& \mathcal{H}_{(1)}^{-}=-\frac{1}{\sqrt{2}} \partial_{1} p^{-}+\frac{1}{\sqrt{2}} \beta \partial_{1}^{2} q^{+}
\end{aligned}
$$


and there is no need of normal ordering. The term $\mathcal{H}_{(a b)}$ is quadratic and contains only the mixed products of $q^{+}, p^{+}$and $q^{-}, p^{-}$

$$
\mathcal{H}_{(a b)}=\frac{1}{2 \beta}: p^{+} p^{-}:+\frac{\beta}{2}: \partial_{1} q^{+} \partial_{1} q^{-}:
$$

The terms $\mathcal{H}_{(a a)}$ and $\mathcal{H}_{(b b)}$ are also quadratic but contain creation and annihilation operators of one kind

$$
\begin{aligned}
\mathcal{H}_{(a a)} & =\frac{1}{2}: p^{+} \partial_{1} q^{+}: \\
\mathcal{H}_{(b b)} & =\frac{1}{2}: p^{-} \partial_{1} q^{-}: .
\end{aligned}
$$

The exponent term

$$
\mathcal{H}_{(\exp )}=\frac{1}{2}: e^{-\sqrt{2}\left(q^{+}+q^{-}\right)}\left[\frac{1}{4 \gamma} P_{\perp}^{2}-\frac{1}{4 \gamma} c_{B}^{2}+\lambda\right]:
$$

is defined by the corresponding Taylor series.

The terms $\mathcal{H}_{(a b)}$ and $\mathcal{H}_{(\exp )}$ are common for $\mathcal{H}^{+}$and $\mathcal{H}^{-}$, while the terms $\mathcal{H}_{(a a)}$ and $\mathcal{H}_{(b b)}$ enter with different signs.

At the classical level the constraints $(77)$ and $(78)$ satisfy the Virasoro algebra. Now the constraints become nonlinear operators and we must compute their quantum algebra. As in the case of ordinary bosonic string we compute the algebra of the Fourier transformed constraints. Using the definition of creation and annihilation operators (54)-(63) one readily finds the explicit expressions for the coordinates and momenta

$$
\begin{aligned}
X^{\mu} & =\frac{1}{\sqrt{2 \pi}} X_{0}^{\mu}+\frac{1}{\sqrt{4 \pi \rho}} \sum_{k=1}^{\infty} \frac{1}{\sqrt{k}}\left[\left(a_{k}^{\mu}+a_{-k}^{* \mu}\right) e^{\imath k \sigma}+\left(a_{-k}^{\mu}+a_{k}^{* \mu}\right) e^{-\imath k \sigma}\right] \\
\Pi^{\mu} & =\frac{1}{\sqrt{2 \pi}} \Pi_{0}^{\mu}+\imath \sqrt{\frac{\rho}{4 \pi}} \sum_{k=1}^{\infty} \sqrt{k}\left[\left(a_{k}^{\mu}-a_{-k}^{* \mu}\right) e^{\imath k \sigma}+\left(a_{-k}^{\mu}-a_{k}^{* \mu}\right) e^{-\imath k \sigma}\right] \\
q^{+} & =\frac{1}{\sqrt{4 \pi \beta}}\left(a_{0}+a_{0}^{*}\right)+\frac{1}{\sqrt{4 \pi \beta}} \sum_{k=1}^{\infty} \frac{1}{\sqrt{k}}\left[\left(a_{k}+a_{-k}^{*}\right) e^{\imath k \sigma}+\left(a_{-k}+a_{k}^{*}\right) e^{-\imath k \sigma}\right] \\
p^{+} & =-\imath \sqrt{\frac{\beta}{4 \pi}}\left(a_{0}-a_{0}^{*}\right)-\imath \sqrt{\frac{\beta}{4 \pi}} \sum_{k=1}^{\infty} \sqrt{k}\left[\left(a_{k}-a_{-k}^{*}\right) e^{\imath k \sigma}+\left(a_{-k}-a_{k}^{*}\right) e^{-\imath k \sigma}\right] \\
q^{-} & =\frac{1}{\sqrt{4 \pi \beta}}\left(b_{0}+b_{0}^{*}\right)+\frac{1}{\sqrt{4 \pi \beta}} \sum_{k=1}^{\infty} \frac{1}{\sqrt{k}}\left[\left(b_{k}+b_{-k}^{*}\right) e^{\imath k \sigma}+\left(b_{-k}+b_{k}^{*}\right) e^{-\imath k \sigma}\right] \\
p^{-} & =\imath \sqrt{\frac{\beta}{4 \pi}}\left(b_{0}-b_{0}^{*}\right)+\imath \sqrt{\frac{\beta}{4 \pi}} \sum_{k=1}^{\infty} \sqrt{k}\left[\left(b_{k}-b_{-k}^{*}\right) e^{\imath k \sigma}+\left(b_{-k}-b_{k}^{*}\right) e^{-\imath k \sigma}\right]
\end{aligned}
$$

We will also need the expression for the nonlocal field

$$
P_{\perp}=\frac{1}{\sqrt{2}} \int_{\sigma_{0}}^{\sigma} d \sigma^{\prime}\left(p^{+}-p^{-}\right)-\frac{\beta}{\sqrt{2}}\left(q^{+}+q^{-}\right)=\frac{1}{\sqrt{2}}\left(r^{+}-r^{-}\right)-\frac{\beta}{\sqrt{2}}\left(q^{+}+q^{-}\right),
$$

entering $\mathcal{H}_{(\exp )}$. Here nonlocal fields $r^{ \pm}=\int_{\sigma_{0}}^{\sigma} d \sigma^{\prime} p^{ \pm}$have the form

$$
r^{+}=-\imath \sqrt{\frac{\beta}{4 \pi}}\left(a_{0}-a_{0}^{*}\right)(\sigma-\tilde{\sigma})
$$




$$
\begin{aligned}
& -\sqrt{\frac{\beta}{4 \pi}} \sum_{k=1}^{\infty} \frac{1}{\sqrt{k}}\left[\left(a_{k}-a_{-k}^{*}\right) e^{\imath k \sigma}-\left(a_{-k}-a_{k}^{*}\right) e^{-\imath k \sigma}\right], \\
r^{-}= & \sqrt{\frac{\beta}{4 \pi}}\left(b_{0}-b_{0}^{*}\right)(\sigma-\tilde{\sigma}) \\
& +\sqrt{\frac{\beta}{4 \pi}} \sum_{k=1}^{\infty} \frac{1}{\sqrt{k}}\left[\left(b_{k}-b_{-k}^{*}\right) e^{\imath k \sigma}-\left(b_{-k}-b_{k}^{*}\right) e^{-\imath k \sigma}\right],
\end{aligned}
$$

where $\tilde{\sigma}$ is the redifined integration constant. We see that nonlocality of the canonical transformation (30) is in fact inessential when considering the theory of closed strings. It enters only through the factor $(\sigma-\tilde{\sigma})$ that does not yield any difficulty in the calculations.

To perform calculations we will widely use the Wick theorem and therefore we introduce the contraction of two linear operators $\varphi$ and $\chi$ as the difference between their product and the corresponding result in normally ordered form

$$
\varphi \chi=\varphi \chi-: \varphi \chi:
$$

Then one easily finds the contractions for nonzero modes

$$
\begin{aligned}
& a_{k} q^{+}=q^{+} \underline{-}_{-k}^{*}=-b_{k} q^{-}=-q^{-} b_{-k}^{*}=\frac{1}{\sqrt{4 \pi \beta k}} e^{-\imath k \sigma}, \\
& a_{-k} q^{+}=q^{+} a_{k}^{*}=-b_{-k} q^{-}=-q^{-} b_{k}^{*}=\frac{1}{\sqrt{4 \pi \beta k}} e^{\imath k \sigma}, \\
& -a_{k} r^{+}=r^{+} \underbrace{*}_{-k}=-b_{k} r^{-}=r^{-} b_{-k}^{*}=\sqrt{\frac{\beta}{4 \pi k}} e^{-\imath k \sigma} \\
& a_{-k} r^{+}=-r^{+} \underline{u}_{k}^{*}=b_{-k} r^{-}=-r^{-} b_{k}^{*}=\sqrt{\frac{\beta}{4 \pi k}} e^{\imath k \sigma},
\end{aligned}
$$

where $k>0$. We will also use the contractions of zero modes

$$
\begin{aligned}
& \underbrace{a^{+}}_{0}=q^{+} \underbrace{*}_{0}=-b_{0} q^{-}=-q^{-} b_{0}^{*}=\frac{1}{\sqrt{4 \pi \beta}} \\
& a_{0} r^{+}=-r^{+} a_{0}^{*}=b_{0} r^{-}=-r^{-} b_{0}^{*}=\imath \sqrt{\frac{\beta}{4 \pi}}(\sigma-\tilde{\sigma}) .
\end{aligned}
$$

The Virasoro generators are equal to the sums of the following operators

$$
\begin{aligned}
L_{X m}^{+} & =\imath \sqrt{\frac{m}{2 \rho}} \Pi_{\mu 0} a_{-m}^{* \mu}+\frac{1}{2} \sum_{k=1}^{m-1} \sqrt{k(m-k)} a_{\mu-k}^{*} a_{-m+k}^{* \mu} \\
& -\sum_{k=1}^{\infty} \sqrt{k(m+k)} a_{\mu-m-k}^{*} a_{-k}^{\mu}, \\
L_{X m}^{-} & =-\imath \sqrt{\frac{m}{2 \rho}} \Pi_{\mu 0} a_{m}^{\mu}+\frac{1}{2} \sum_{k=1}^{m-1} \sqrt{k(m-k)} a_{\mu k} a_{m-k}^{\mu} \\
& -\sum_{k=1}^{\infty} \sqrt{k(m+k)} a_{\mu k}^{*} a_{m+k}^{\mu}, \\
L_{(1) m}^{+} & =m \sqrt{\frac{\pi \beta m}{2}}\left(a_{m}-a_{-m}^{*}-b_{m}-b_{-m}^{*}\right),
\end{aligned}
$$




$$
\begin{aligned}
& \left.L_{(1) m}^{-}=-m \sqrt{\frac{\pi \beta m}{2}}\left(a_{m}+a_{-m}^{*}-b_{m}+b_{-m}^{*}\right)\right] \\
& L_{(a b) m}=\frac{\sqrt{m}}{4}\left(a_{0} b_{m}+a_{m} b_{0}-a_{0}^{*} b_{m}-a_{-m}^{*} b_{0}-b_{0}^{*} a_{m}-b_{-m}^{*} a_{0}+a_{0}^{*} b_{-m}^{*}+a_{-m}^{*} b_{0}^{*}\right) \\
& -\frac{1}{2} \sum_{k=1}^{m-1} \sqrt{k(m-k)}\left(a_{-k}^{*} b_{m-k}+b_{-k}^{*} a_{m-k}\right) \\
& +\frac{1}{2} \sum_{k=1}^{\infty} \sqrt{k(m+k)}\left(a_{-k} b_{m+k}+a_{m+k} b_{-k}+a_{k}^{*} b_{-m-k}^{*}+a_{-m-k}^{*} b_{k}^{*}\right), \\
& L_{(a a) m}=\frac{\sqrt{m}}{4}\left(a_{0} a_{m}-a_{0}^{*} a_{m}+a_{-m}^{*} a_{0}-a_{0}^{*} a_{-m}^{*}\right) \\
& +\frac{1}{4} \sum_{k=1}^{m-1} \sqrt{k(m-k)}\left(a_{k} a_{m-k}-a_{-k}^{*} a_{-m+k}^{*}\right) \\
& +\frac{1}{2} \sum_{k=1}^{\infty} \sqrt{k(m+k)}\left(a_{-m-k}^{*} a_{-k}-a_{k}^{*} a_{m+k}\right), \\
& L_{(b b) m}=-\frac{\sqrt{m}}{4}\left(b_{0} b_{m}-b_{0}^{*} b_{m}+b_{-m}^{*} b_{0}-b_{0}^{*} b_{-m}^{*}\right) \\
& -\frac{1}{4} \sum_{k=1}^{m-1} \sqrt{k(m-k)}\left(b_{k} b_{m-k}-b_{-k}^{*} b_{-m+k}^{*}\right) \\
& -\frac{1}{2} \sum_{k=1}^{\infty} \sqrt{k(m+k)}\left(b_{-m-k}^{*} b_{-k}-b_{k}^{*} b_{m+k}\right), \\
& L_{(e x p) m}=\int_{-\pi}^{\pi} d \sigma: \mathcal{H}_{e x p}: e^{-\imath m \sigma} .
\end{aligned}
$$

The last expression for $L_{(\exp ) m}$ cannot be written as the sum of creation and annihilation operators in compact form. Nevertherless calculations can be easily performed in the integral representation.

For $m=1$ finite sums in (100), (101), (104), (105), and (106) are absent.

For $m=0$ the Virasoro constraints have the form

$$
\begin{aligned}
L_{0}^{+}= & -\frac{1}{4 \rho} \Pi_{\mu 0} \Pi_{0}^{\mu}-\sum_{k=1}^{\infty} k a_{\mu-k}^{*} a_{-k}^{\mu}+\frac{1}{4}\left(a_{0} b_{0}-a_{0}^{*} b_{0}-b_{0}^{*} a_{0}+a_{0}^{*} b_{0}^{*}\right) \\
& +\frac{1}{2} \sum_{k=1}^{\infty} k\left(a_{-k} b_{k}+a_{k} b_{-k}+a_{k}^{*} b_{-k}^{*}+a_{-k}^{*} b_{k}^{*}\right) \\
& -\frac{1}{2} \sum_{k=1}^{\infty} k\left(a_{k}^{*} a_{k}-a_{-k}^{*} a_{-k}-b_{k}^{*} b_{k}+b_{-k}^{*} b_{-k}\right)+\int_{-\pi}^{\pi} d \sigma: \mathcal{H}_{(\exp )}: \\
L_{0}^{-}= & -\frac{1}{4 \rho} \Pi_{\mu 0} \Pi_{0}^{\mu}-\sum_{k=1}^{\infty} k a_{\mu k}^{*} a_{k}^{\mu}+\frac{1}{4}\left(a_{0} b_{0}-a_{0}^{*} b_{0}-b_{0}^{*} a_{0}+a_{0}^{*} b_{0}^{*}\right) \\
& +\frac{1}{2} \sum_{k=1}^{\infty} k\left(a_{-k} b_{k}+a_{k} b_{-k}+a_{k}^{*} b_{-k}^{*}+a_{-k}^{*} b_{k}^{*}\right) \\
& +\frac{1}{2} \sum_{k=1}^{\infty} k\left(a_{k}^{*} a_{k}-a_{-k}^{*} a_{-k}-b_{k}^{*} b_{k}+b_{-k}^{*} b_{-k}\right)+\int_{-\pi}^{\pi} d \sigma: \mathcal{H}_{(\exp )}:
\end{aligned}
$$

Of course, arbitrary constants can be added to $L_{0}^{ \pm}$due to an ambiquity in the operator ordering. We drop this possibility at present because we will not discuss in detail the 
physical interpretation of the Fock space in the present paper. The constraint $L_{0}$ then reads

$$
\begin{aligned}
L_{0} & =L_{0}^{+}+L_{0}^{-}=-\frac{1}{2 \rho} \Pi_{\mu 0} \Pi_{0}^{\mu}-\sum_{k=1}^{\infty} k\left(a_{\mu k}^{*} a_{k}^{\mu}+a_{\mu-k}^{*} a_{-k}^{\mu}\right) \\
& +\frac{1}{2}\left(a_{0} b_{0}-a_{0}^{*} b_{0}-b_{0}^{*} a_{0}+a_{0}^{*} b_{0}^{*}\right) \\
& +\sum_{k=1}^{\infty} k\left(a_{-k} b_{k}+a_{k} b_{-k}+a_{k}^{*} b_{-k}^{*}+a_{-k}^{*} b_{k}^{*}\right)+2 \int_{-\pi}^{\pi} d \sigma: \mathcal{H}_{(\exp )}:
\end{aligned}
$$

Its quadratic part corresponding to $q^{ \pm}$is undiagonal and yields the problem of interpretation of the states. We briefly discuss this point at the end of Section 3.6. Here we note that the constructed Fock space contains two unphysical modes besides the physical ones and $L_{0}$ is not the energy of the string but just the constraint. Therefore one has no reason for the diagonality of $L_{0}$.

Expressions for the operators with negative $m<0$ are easily obtained from those of positive $m>0$ by Hermitian conjugation

$$
L_{-m}=\left(L_{m}\right)^{*}
$$

They are not written down explicitly for the sake of space.

So we got expressions for all terms entering the Virasoro constraints. The terms $L_{(1) m}^{ \pm}$ are linear, while $L_{X m}^{ \pm}, L_{(a b) m}, L_{(a a) m}, L_{(b b) m}$ are belinear in creation and annihilation operators. The exponent term $L_{(\exp ) m}$ is nonpolinomial and is understood as the normally ordered power series.

\subsection{General remark}

Here we make the general remark concerning calculations of commutators of quantum operators. Suppose we have two polynomial operators $: p_{n}^{N} q_{m}^{M}$ : and $: p_{k}^{K} q_{l}^{L}$ : written as the normally ordered product on canonical coordinates $q_{m}, q_{l}$ and conjugate momenta $p_{n}$, $p_{k}$. Here $N, M, K$, and $L$ denote the corresponding powers.

Classical expressions of these operators have the following Poisson bracket

$$
\left\{p_{n}^{N} q_{m}^{M}, p_{k}^{K} q_{l}^{L}\right\}=M K p_{n}^{N} q_{m}^{M-1} p_{k}^{K-1} q_{l}^{L} \delta_{m,-k}-N L p_{n}^{N-1} q_{m}^{M} p_{k}^{K} q_{l}^{L-1} \delta_{n,-l},
$$

where for definitness we used the basic Poisson brackets (52).

Let us consider the commutator

$$
\left[: p_{n}^{N} q_{m}^{M}:,: p_{k}^{K} q_{l}^{L}:\right]
$$

Due to the Wick theorem the right hand side of this commutator contains normal products with zero number of contraction, single contractions, double contractions, and so on. If there were no contractions then the operators would commute. If there were only single contractions then one would obtain exactly the classic result multiplied by $\imath$. Indeed, consider all terms in the right hand side of (109) containing single contractions

$$
\left[: p_{n}^{N} q_{m}^{M}:,: p_{k}^{K} q_{l}^{L}:\right] \stackrel{(1)}{=} N K: p_{n}^{N-1} q_{m}^{M} p_{k}^{K-1} q_{l}^{L}:\left(p_{n} p_{k}-p_{k} p_{n}\right)
$$




$$
\begin{aligned}
& +N L: p_{n}^{N-1} q_{m}^{M} p_{k}^{K} q_{l}^{L-1}:\left(p_{n} q_{l}-q_{l} p_{n}\right) \\
& +M K: p_{n}^{N} q_{m}^{M-1} p_{k}^{K-1} q_{l}^{L}:\left(q_{m} p_{k}-p_{k} q_{m}\right) \\
& +M L: p_{n}^{N} q_{m}^{M-1} p_{k}^{K} q_{l}^{L-1}:\left(q_{m} q_{l}-q_{l} q_{m}\right)
\end{aligned}
$$

where superscript (1) denotes that only single contractions are used in the Wick theorem and where elementary contractions are given by

$$
\begin{aligned}
p_{n} p_{k} & =\frac{\beta|n|}{2} \delta_{n,-k}, \\
p_{n} q_{l} & =-\frac{\imath}{2} \delta_{n,-l}, \\
q_{l}^{p_{n}} & =\frac{\imath}{2} \delta_{n,-l}, \\
q_{m} q_{l} & =\frac{1}{2 \beta|m|} \delta_{m,-l}
\end{aligned}
$$

For definitness but without loss of generality we consider here the contractions for $q_{k}^{+}$and $p_{k}^{+}(66),(67)$. Then the first and the last terms in (110) dissappear and one is left with the classic result

$$
\left[: p_{n}^{N} q_{m}^{M}:,: p_{k}^{K} q_{l}^{L}:\right] \stackrel{(1)}{=} \imath M K: p_{n}^{N} q_{m}^{M-1} p_{k}^{K-1} q_{l}^{L}: \delta_{m,-k}-\imath N L: p_{n}^{N-1} q_{m}^{M} p_{k}^{K} q_{l}^{L-1}: \delta_{n,-l} .
$$

It is clear that no extra terms as compared to the classical result appear if only single contractions are taken into account. Thus single contractions exactly reproduce the classical Poisson bracket relations up to the factor $\imath$. This result is readily generalised to arbitrary polinomials in $q, p$.

The terms containing two or more contractions yield the quantum corrections. If at the classical level some set of nonlinear operators obeyed the closed Poisson bracket algebra, then at the quantum level this algebra could be broken by the quantum corrections producing the anomaly. A set of first class constraints reflects the gauge symmetry of the action and the existence of anomaly means that the symmetry is broken at the quantum level. The famous example of such a theory is the ordinary bosonic string where the conformal symmetry is broken by the central charge of the Virasoro algebra arising from double contractions in the Wick theorem. In critical dimension $D=26$ the central charge can be compensated in the enlarged Fock space by ghost modes.

To compute the anomaly we must consider only the terms containing two or more contractions. In the following sections we show that in spite of the nonpolinomial constraints in the theory of the string with dynamical geometry the only anomaly is the anomaly of ordinary bosonic string. The computation will be devided in two parts. First, we will consider the diagonal terms and next the crossterms in the commutators.

\subsection{Diagonal terms}

Let us consider the commutators of $L_{X m}^{ \pm}, L_{(a b) m}, L_{(a a) m}, L_{(b b) m}$, and $L_{(e x p) m}$ with themselves. The operators $L_{(1) m}^{ \pm}$are linear and due to the general remark their commutators produce no anomalous terms. Therefore they are not considered in what follows. 
Consider the commutator

$$
\left[L_{X m}^{+}, L_{X n}^{+}\right]
$$

The terms containing one creation and one annihilation operators do not produce any anomaly because one of the two contractions is always zero. So double contractions can arise only within the terms included in the finite sum (100). For $m, n \geq 0$ there are no double contractions and therefore the anomaly is absent. The commutator

$$
\left[L_{X m}^{+}, L_{X-n}^{+}\right]
$$

where $m, n>0$ does contain double contractions arising from the finite sums

$$
\begin{aligned}
{\left[L_{X m}^{+}, L_{X-n}^{+}\right] } & \stackrel{(2)}{=} \frac{1}{4}\left[\sum_{k=1}^{m-1} \sqrt{k(m-k)} a_{\mu-k}^{*} a_{-m+k}^{* \mu}, \sum_{l=1}^{n-1} \sqrt{l(n-l)} a_{\nu-l} a_{-n+l}^{\nu}\right] \\
& \stackrel{(2)}{=}-\frac{1}{4} \sum_{k=1}^{m-1} \sum_{l=1}^{n-1} \sqrt{k(m-k) l(n-l)}\left[a_{\nu-l} a_{\mu-k}^{*} a_{-n+l}^{\nu} a_{-m+k}^{* \mu}+a_{\nu-l} a_{-m+k}^{* \mu} a_{-n+l}^{\nu} a_{\mu-k}^{*}\right] \\
& =-\frac{D}{2} \sum_{k=1}^{m-1} k(m-k) \delta_{m, n}=-\frac{D}{12} m\left(m^{2}-1\right) \delta_{m, n}
\end{aligned}
$$

This is the famous expression for the central charge in the Virasoro algebra. The zero modes do not change its value irrespective of their normal ordering prescription because $L_{X m}^{+}$does not contain $X_{0}^{\mu}$.

The same calculations yield the central charge in the commutator

$$
\left[L_{X m}^{-}, L_{X-n}^{-}\right] \stackrel{(2)}{=} \frac{D}{12} m\left(m^{2}-1\right) \delta_{m, n}
$$

It differs from (111) only by the sign because nontrivial contractions arise when $L_{X m}^{-}$ stands on the left.

In contrast to the $L_{X m}^{ \pm}$the finite sum in $L_{(a b) m}$ does not yield any contribution to the anomaly. The suspicious terms are only those containg zero modes and entering the infinite sum. For $m, n \geq 0$, the anomalous terms in the commutator

$$
\left[L_{(a b) m}, L_{(a b) n}\right]
$$

are clearly absent. Nontrivial calculations occur only in the commutator

$$
\begin{aligned}
& {\left[L_{(a b) m}, L_{(a b)-n}\right] \stackrel{(2)}{=} \frac{\sqrt{m n}}{16}\left[a_{0} a_{0}^{*} b_{m} b_{n}^{*}+b_{0} b_{0}^{*} a_{m} a_{n}^{*}-a_{0} a_{0}^{*} b_{-n} b_{-m}^{*}-b_{0} b_{0}^{*} a_{-n} a_{-m}^{*}\right]} \\
& +\frac{1}{4} \sum_{k, l=1}^{\infty} \sqrt{k(m+k) l(n+l)} \\
& \times[a_{-k} a_{-l}^{*} b_{m+k} b_{n+l}^{*}+a_{m+k} a_{n+l}^{*} b_{-k} b_{-l}^{*}-\underbrace{}_{l} a_{k}^{*} b_{-n-l} b_{-m-k}^{*}-a_{-n-l} a_{-m-k}^{*} b_{l} b_{k}^{*}] \\
& =\frac{m}{16}\left(-2 \delta_{m, n}+2 \delta_{m, n}\right)+\frac{1}{4} \sum_{k=1}^{\infty} k(m+k)\left(-2 \delta_{m, n}+2 \delta_{m, n}\right)=0 .
\end{aligned}
$$

So all anomalous terms cancell. This happened because the operator $L_{(a b) m}$ contains terms quadratic in creation operators along with the terms quadratic in annihilation operators. 
The terms yielding nontrivial double contractions in $L_{(a a) m}$ are those containing the zero mode and entering the finite sum. Nonzero double contractions arise only in the commutator

$$
\begin{aligned}
& {\left[L_{(a a) m}, L_{(a a)-n}\right] \stackrel{(2)}{=} \frac{\sqrt{m n}}{16}\left[a_{0} a_{0}^{*} a_{m} a_{n}^{*}-a_{0} a_{0}^{*} a_{-n} a_{-m}^{*}\right]} \\
& +\frac{1}{4} \sum_{k=1}^{m-1} \sum_{l=1}^{n-1} \sqrt{k(m-k) l(n-l)}[\underbrace{}_{k} a_{l}^{*} a_{m-k} a_{n-l}^{*}-a_{-l} a_{-k}^{*} a_{-n+l} a_{-m+k}^{*}] \\
& =\frac{m}{16}\left(\delta_{m, n}-\delta_{m, n}\right)+\frac{1}{4} \sum_{k=1}^{m-1} k(m-k)\left(\delta_{m, n}-\delta_{m, n}\right)=0,
\end{aligned}
$$

where $m, n>0$. Again all anomalous terms cancell.

Similar calculations show that all anomalous terms in the diagonal commutator

$$
\left[L_{(b b) m}, L_{(b b) n}\right]
$$

cancell for all values of $m$ and $n$. We omit here the details for the sake of space.

The anomalous terms do not appear in the commutator

$$
\left[L_{(e x p) m}, L_{(e x p) n}\right]
$$

for another reason. Here one encounts infinite number of contractions but all off them are zero. Indeed, operator $L_{(e x p) m}$ depends only from two combinations of the fields $q^{+}+q^{-}$ and $p^{+}-p^{-}$. Easy analysis show that

$$
\left(q^{+}+q^{-}\right)\left(q^{+}+q^{-}\right)=\left(q^{+}+q^{-}\right)\left(p^{+}-p^{-}\right)=\left(p^{+}-p^{-}\right)\left(p^{+}-p^{-}\right)=0 .
$$

Thus the exponential terms clearly commute due to the Wick theorem. The essential point here is that the creation operators for $q^{+}$and $q^{-}$produce the states of opposite norm. Only in this case contractions (112) equal zero.

So we see the difference between the quantum Liouville theory and quantum twodimensional gravity with torsion. Instead of one field the latter theory contains a pair of fields which contribute to the energy with opposite signs. These fields can be arranged in such a way that the exponents commute. In this way one of the main trouble of quantum Liouville theory is avoided.

\subsection{Crossterms}

To compute the crossterms commutators one should perform more tedious calculations as compared to the diagonal commutators. It is clear that $L_{X m}^{+}$commute with $L_{X m}^{-}$and both of them commute with the operators $L_{(a b) m}, L_{(a a) m}, L_{(b b) m}$, and $L_{(e x p) m}$. The crossterms commutators

$$
\left[L_{(a b) m}, L_{(a a) n}\right] \stackrel{(2)}{=}\left[L_{(a b) m}, L_{(b b) n}\right] \stackrel{(2)}{=}\left[L_{(a a) m}, L_{(b b) n}\right] \stackrel{(2)}{=} 0
$$

also yield no anomaly because there are unsufficient number of creation and annihilation operators of the same kind. So we have to consider the crossterms between $L_{(a b) m}, L_{(a a) m}$, $L_{(b b) m}$ and the exponent term $L_{(e x p) m}$. Here we must consider only double contractions because one of the operators is necessarily quadratic. 
Let us consider the crossterms

$$
\left[L_{(a b) m}, L_{(\exp ) n}\right]+\left[L_{(e x p) m}, L_{(a b) n}\right]=\left[L_{(a b) m}, L_{(\exp ) n}\right]-(m \leftrightarrow n)
$$

which enter the commutator $\left[L_{m}^{+}, L_{n}^{+}\right]$precisely in this combination. To show how the cancellation occurs we consider first the commutators of the terms containing the zero modes in $L_{(a b) m}$ and the cosmological term in $L_{(\exp ) n}$

$$
\left[\frac{\sqrt{m}}{4}\left(a_{0} b_{m}+a_{m} b_{0}+a_{0}^{*} b_{-m}^{*}+a_{-m}^{*} b_{0}^{*}\right), \frac{\lambda}{2} \int_{-\pi}^{\pi} d \sigma: e^{-\sqrt{2}\left(q^{+}+q^{-}\right)}: e^{-\imath n \sigma}\right]-(m \leftrightarrow n) .
$$

Expanding the operator exponent in a Taylor series, taking all possible double contractions and then gathering the exponent again one obtains

$$
\begin{aligned}
& \frac{\lambda \sqrt{m}}{4} \int_{-\pi}^{\pi} d \sigma: e^{-\sqrt{2}\left(q^{+}+q^{-}\right)}: e^{-\imath n \sigma} \\
& \times\left[a_{0} q^{+} b_{m} q^{-}+a_{m} q^{+} b_{0} q^{-}-q^{+} a_{0}^{*} q^{-} b_{-m}^{*}-q^{+} a_{-m}^{*} q^{-} b_{0}^{*}\right]-(m \leftrightarrow n) \\
& =\frac{\lambda}{4} \int_{-\pi}^{\pi} d \sigma: e^{-\sqrt{2}\left(q^{+}+q^{-}\right)}: e^{-\imath n \sigma}\left[-\frac{1}{4 \pi \beta} e^{-\imath m \sigma}+\frac{1}{4 \pi \beta} e^{-\imath m \sigma}\right]-(m \leftrightarrow n)=0,
\end{aligned}
$$

where contractions (94)-(99) have been used. So the cancellation occurs inside the square brackets in the last expression before the antisymmetrization. Computing the commutator of quadratic terms containing zero modes in $L_{(a b) m}$ with whole exponential term one must also take into account the double contractions with $\left(r^{+}-r^{-}\right)$and double mixed contractions: the first with $\left(q^{+}+q^{-}\right)$and the second with $\left(r^{+}-r^{-}\right)$. Double contractions with $\left(q^{+}+q^{-}\right)$when one factor $\left(q^{+}+q^{-}\right)$is taken from the exponent and the other is taken from $P_{\perp}^{2}$ term cancell like in the previous case. Double contractions with $\left(r^{+}-r^{-}\right)$ cancell precisely in the same way as those with $\left(q^{+}+q^{-}\right)$. Double mixed contractions cancell in another way. That is, computing the commutator (113) one obtains the term

$$
\begin{aligned}
& \sqrt{m} \int_{-\pi}^{\pi} d \sigma: e^{-\sqrt{2}\left(q^{+}-q^{-}\right)}\left(P_{\perp}-c_{B}\right): e^{-\imath n \sigma} \\
& \times\left[-a_{0} q^{+} b_{m} r^{-}+a_{0} r^{+} b_{m} q^{-}-a_{m} q^{+} b_{0} r^{-}+a_{m} r^{+} b_{0} q^{-}\right. \\
& \left.+q^{+} a_{0}^{*} r^{-} b_{-m}^{*}-r^{+} a_{0}^{*} q^{-} b_{-m}^{*}+q^{+} a_{-m}^{*} r^{-} b_{0}^{*}-r^{+} a_{-m}^{*} q^{-} b_{0}^{*}\right]-(m \leftrightarrow n) \\
& =\int_{-\pi}^{\pi} d \sigma: e^{-\sqrt{2}\left(q^{+}+q^{-}\right)}\left(P_{\perp}-c_{B}\right):\left[\frac{1}{\pi}-\frac{\imath}{\pi}(\sigma-\tilde{\sigma})\right] e^{-\imath(m+n) \sigma}-(m \leftrightarrow n)=0,
\end{aligned}
$$

where we have dropped insignificant constant factor. It is cancelled after antisymmetrization in $m, n$. So we have proved that double contractions of the first term in $L_{(a b) m}$ with $L_{(\exp ) n}$ produce no anomaly. Now we are left to consider double contractions of the infinite sum in (104) because finite sum can yield only single nontrivial contractions. Using contractions (94)-(97) one easily shows that all double contractions with $\left(q^{+}+q^{-}\right)^{2}$, $\left(q^{+}+q^{-}\right)\left(r^{+}-r^{-}\right)$, and $\left(r^{+}-r^{-}\right)^{2}$ cancell. We omit the corresponding calculations. Thus for $m, n>0$ the crossterm commutator equals zero

$$
\left[L_{(a b) m}, L_{(e x p) n}\right]-(m \leftrightarrow n)=0 .
$$


This is the exact result because single contractions also yield zero as follows from Poisson brackets calculation. One can check by similar calculations that this result holds for all values of $m$ and $n$.

Let us consider the next crossterms commutator

$$
\left[L_{(a a) m}, L_{(\exp ) n}\right]-(m \leftrightarrow n)
$$

for $m, n>0$. The first term in $L_{(a a) m}$ containing the zero mode produce the following double contractions with $\left(q^{+}+q^{-}\right)^{2},\left(q^{+}+q^{-}\right)\left(r^{+}-r^{-}\right)$, and $\left(r^{+}-r^{-}\right)^{2}$

$$
\begin{aligned}
& \sqrt{m}\left[a_{0} q^{+} a_{m} q^{+}+q^{+} a_{0}^{*} q^{+} a_{-m}^{*}\right] e^{-\imath n \sigma}-(m \leftrightarrow n)=\frac{1}{4 \pi \beta} e^{-\imath(m+n) \sigma}-(m \leftrightarrow n)=0, \\
& \sqrt{m}\left[a_{0} q^{+} a_{m} r^{+}+a_{0} r^{+} a_{m} q^{+}+q^{+} a_{0}^{*} r^{+} a_{-m}^{*}+r^{+} a_{0}^{*} q^{+} a_{-m}^{*}\right] e^{-\imath n \sigma}-(m \leftrightarrow n) \\
& =\left[-\frac{1}{4 \pi}+\imath \frac{\sigma-\tilde{\sigma}}{4 \pi}+\frac{1}{4 \pi}-\imath \frac{\sigma-\tilde{\sigma}}{4 \pi}\right] e^{-\imath(m+n) \sigma}-(m \leftrightarrow n)=0, \\
& \sqrt{m}\left[a_{0} r^{+} a_{m} r^{+}+r^{+} a_{0}^{*} r^{+} a_{-m}^{*}\right] e^{-\imath n \sigma}-(m \leftrightarrow n) \\
& =-\imath \frac{\beta(\sigma-\tilde{\sigma})}{2 \pi} e^{-\imath(m+n) \sigma}-(m \leftrightarrow n)=0,
\end{aligned}
$$

and all of them cancell. Here we omit the integration over $\sigma$ because cancellation occures under the integral sign and drop constant factors. Next we have to consider double contractions between the finite sum in $L_{(a a) m}$ and $L_{(e x p) n}$. Here we must be carefull because the upper limit of summation depends on $m$. Straightforward calculations show that two terms arising from double contractions with $\left(q^{+}+q^{-}\right)^{2},\left(q^{+}+q^{-}\right)\left(r^{+}-r^{-}\right)$, and $\left(r^{+}-r^{-}\right)^{2}$ survive

$$
\begin{aligned}
& \sum_{k=1}^{m-1} \sqrt{k(m-k)}\left[a_{k} q^{+} a_{m-k} q^{+}+q^{+} a_{-k}^{*} q^{+} a_{-m+k}^{*}\right] e^{-\imath n \sigma}-(m \leftrightarrow n) \\
& =\sum_{k=1}^{m-1} \frac{1}{2 \pi \beta} e^{-\imath(m+n) \sigma}-(m \leftrightarrow n) \neq 0, \\
& \sum_{k=1}^{m-1} \sqrt{k(m-k)}\left[a_{k} q^{+} a_{m-k} r^{+}+q^{+} a_{-k}^{*} r^{+} a_{-m+k}^{*}\right] e^{-\imath n \sigma}-(m \leftrightarrow n) \\
& =\sum_{k=1}^{m-1}\left(-\frac{1}{4 \pi}+\frac{1}{4 \pi}\right) e^{-\imath(m+n) \sigma}-(m \leftrightarrow n)=0, \\
& \sum_{k=1}^{m-1} \sqrt{k(m-k)}\left[a_{k} r^{+} a_{m-k} r^{+}+r^{+} a_{-k}^{*} r^{+} a_{-m+k}^{*}\right] e^{-\imath n \sigma}-(m \leftrightarrow n) \\
& =\sum_{k=1}^{m-1} \frac{\beta}{2 \pi} e^{-\imath(m+n) \sigma}-(m \leftrightarrow n) \neq 0 .
\end{aligned}
$$

Here for brevity we also do not write the integral over $\sigma$. The first and the third terms do not cancell because of the upper limit in the sum. So we have considered all double contractions in the crossterms commutator (114) and they lead to a nonzero result.

Let us consider the last crossterms commutator

$$
\left[L_{(b b) m}, L_{(e x p) n}\right]-(m \leftrightarrow n)
$$


for $m, n>0$. Calculations in this case are very similar to the previous ones. We obtain precisely the same results with one very important difference. The finite sum in $L_{(b b) m}$ has opposite sign to that of $L_{(a a) m}$ and one obtains the terms which exactly cancell (115) and (117). So we have proved that for $m, n>0$ the crossterms commutator

$$
\left[L_{(a a) m}+L_{(b b) m}, L_{(e x p) n}\right]-(m \leftrightarrow n)=0
$$

equals zero. This results can be generalised for all values of $m$ and $n$ by direct calculations which are omitted here.

Thus we have proved that all double contractions in crossterms commutators cancell and there is no quantum corrections to the classical algebra of the constraints in pure twodimensional gravity with torsion. The only anomaly comes from the string coordinates, and the quantum algebra of the constraints coincides with that of ordinary bosonic string

$$
\begin{aligned}
& {\left[L_{m}^{+}, L_{n}^{+}\right]=-(m-n) L_{m+n}^{+}-\frac{D}{12} m\left(m^{2}-1\right) \delta_{m,-n}} \\
& {\left[L_{m}^{+}, L_{n}^{-}\right]=0} \\
& {\left[L_{m}^{-}, L_{n}^{-}\right]=(m-n) L_{m+n}^{-}+\frac{D}{12} m\left(m^{2}-1\right) \delta_{m,-n}}
\end{aligned}
$$

So the central charge in the Virasoro algebra of the string with dynamical geometry precisely coinside with that of an ordinary bosonic string if we use the standart representation for the string coordinates. In the following section we construct new Fock representation for a bosonic string where the value of the central charge differs by two.

\subsection{Anomaly free string in two dimensions}

The canonical transformation converting $q, p$ and $q_{\perp}, p_{\perp}$ into $q^{ \pm}, p^{ \pm}$before the introduction of creation and annihilation operators is a crucial point for the construction of the anomaly free representation of the Virasoro algebra for pure two-dimensional gravity with torsion. If we constructed the Fock space directly from $q, p$ and $q_{\perp}, p_{\perp}$ then we would obtain the anomalous theory. There would arise not only the central charge but the algebra would be completely broken. For example, the exponential terms would produce other exponential terms with different exponents. As a by-product of our construction we proved that in the Fock space for $q^{ \pm}, p^{ \pm}$the central charge produced by quadratic terms in (47) and (48) which have precisely the same form as the constraints for string coordinates does not appear at the quantum level. This sugests new representation for an ordinary bosonic string.

Let us make the canonical transformation to the light cone variables

$$
X^{0}, X^{1}, \Pi_{0}, \Pi_{1} \rightarrow X^{ \pm}, \Pi^{ \pm}
$$

with the generating function

$$
F_{5}=-\frac{1}{\sqrt{2}}\left(X^{+}-X^{-}\right) \Pi_{0}-\frac{1}{\sqrt{2}}\left(X^{+}+X^{-}\right) \Pi_{1}
$$

depending on new coordinates and old momenta. Other string coordinates remain unchanged. New coordinates and momenta are related to the old ones by the formulas

$$
X^{+}=\frac{1}{\sqrt{2}}\left(X^{1}+X^{0}\right), \quad \Pi^{+}=\frac{1}{\sqrt{2}}\left(\Pi_{1}+\Pi_{0}\right),
$$




$$
X^{-}=\frac{1}{\sqrt{2}}\left(X^{1}-X^{0}\right), \quad \Pi^{-}=\frac{1}{\sqrt{2}}\left(\Pi_{1}-\Pi_{0}\right) .
$$

We introduce annihilation and creation operators $c, d$ and $c^{*}, d^{*}$ for the Fourier transformed light cone variables

$$
\begin{array}{ll}
X_{k}^{+}=\frac{1}{\sqrt{2 \rho|k|}}\left(d_{k}+d_{-k}^{*}\right), & X_{k}^{-}=\frac{1}{\sqrt{2 \rho|k|}}\left(c_{k}+c_{-k}^{*}\right), \\
\Pi_{k}^{+}=-\imath \sqrt{\frac{\rho|k|}{2}}\left(d_{k}-d_{-k}^{*}\right), & \Pi_{k}^{-}=\imath \sqrt{\frac{\rho|k|}{2}}\left(c_{k}-c_{-k}^{*}\right),
\end{array}
$$

where $k \neq 0$. They satisfy canonical commutation relations

$$
-\left[c_{k}, c_{l}^{*}\right]=\left[d_{k}, d_{l}^{*}\right]=\delta_{k, l}
$$

and are related to the old creation and annihilation operators (54), (55) by linear transformation

$$
\begin{array}{rlrl}
c_{k} & =\frac{1}{\sqrt{2}}\left(a_{-k}^{* 1}-a_{k}^{0}\right), & d_{k}=\frac{1}{\sqrt{2}}\left(a_{k}^{1}+a_{-k}^{* 0}\right), \\
c_{k}^{*}=\frac{1}{\sqrt{2}}\left(a_{-k}^{1}-a_{k}^{* 0}\right), & d_{k}^{*}=\frac{1}{\sqrt{2}}\left(a_{k}^{* 1}+a_{-k}^{0}\right) .
\end{array}
$$

Here important point is that the canonical commutation relations of $c_{k}$ and $c_{k}^{*}$ have negative sign as well as that of $a_{k}^{0}$ and $a_{k}^{* 0}$.

In the present section for brevity we omit the zero modes because they do not contribute to the central charge.

In terms of new creation and annihilation operators the $X^{0}, X^{1}$ part of the constraints for $m>0$ up to zero modes read

$$
L_{X m}^{ \pm}=L_{(c d) m} \pm\left(L_{(c c) m}+L_{(d d) m}\right)
$$

where

$$
\begin{aligned}
L_{(c d) m} & =-\frac{1}{2} \sum_{k=1}^{m-1} \sqrt{k(m-k)}\left(d_{-k}^{*} c_{m-k}+c_{-k}^{*} d_{m-k}\right) \\
& +\frac{1}{2} \sum_{k=1}^{\infty} \sqrt{k(m+k)}\left(d_{-k} c_{m+k}+d_{m+k} c_{-k}+d_{k}^{*} c_{-m-k}^{*}+d_{-m-k}^{*} c_{k}^{*}\right) \\
L_{(c c) m} & =-\frac{1}{4} \sum_{k=1}^{m-1} \sqrt{k(m-k)}\left(c_{k} c_{m-k}-c_{-k}^{*} c_{-m+k}^{*}\right) \\
& -\frac{1}{2} \sum_{k=1}^{\infty} \sqrt{k(m+k)}\left(c_{-m-k}^{*} c_{-k}-c_{k}^{*} c_{m+k}\right) \\
& =\frac{1}{4} \sum_{k=1}^{m-1} \sqrt{k(m-k)}\left(d_{k} d_{m-k}-d_{-k}^{*} d_{-m+k}^{*}\right) \\
& +\frac{1}{2} \sum_{k=1}^{\infty} \sqrt{k(m+k)}\left(d_{-m-k}^{*} d_{-k}-d_{k}^{*} d_{m+k}\right) .
\end{aligned}
$$


This form of the constraints have precisely the same form as $L_{(a b)} \pm\left(L_{(a a)}+L_{(b b)}\right)$ after replacement $a, b \rightarrow d, c$. Therefore formal commutators have no anomalous term as compared to the classical Poisson brackets.

This is an intriguing observation, but we must pay for the absence of the central charge. In fact, the constraints (125)-(127) are not well defined on the basis of the new Fock space because they produce the states with infinite norm. So they are unbounded operators and one must be carefull to give a precise meaning of the commutators. At present the calculations must be considered only as the formal manipulations with the operator symbols. We hope that there exists more rigorous foundation of the present calculations.

In contrast to the ordinary representation one can impose the whole set of quantum constraints on the physical subspace

$$
L_{m}^{ \pm} \mid p h y s>=0
$$

for all $m$. At the formal level the physical space is not empty. For example, the state

$$
\mid p h>=\exp \left[ \pm \sum_{l=1}^{\infty}\left(c_{l}^{*} c_{-l}^{*}+d_{l}^{*} d_{-l}^{*}\right] \mid 0>\right.
$$

is annihilated by all $L_{X m}^{ \pm}$

$$
L_{(c d) m}\left|p h>=L_{(c c) m}\right| p h>=L_{(d d) m} \mid p h>=0 .
$$

It has infinite norm and its interpretation is obscure. But the mere fact of its existence seems to be interesting.

One may try also to find new Fock representation for the remaining space components of the string by introducing complex string coordinates like $X^{2} \pm \imath X^{3}$ and corresponding creation and annihilation operators. Unfortunately, this approach does not solve the problem of anomaly. This shows that different signs in the canonical commutation relations (121) are of prime importance.

In the new representation the quantum Virasoro algebra has the form

$$
\begin{aligned}
& {\left[L_{m}^{+}, L_{n}^{+}\right]=-(m-n) L_{m+n}^{+}-\frac{D-2}{12} m\left(m^{2}-1\right) \delta_{m,-n}} \\
& {\left[L_{m}^{+}, L_{n}^{-}\right]=0} \\
& {\left[L_{m}^{-}, L_{n}^{-}\right]=(m-n) L_{m+n}^{-}+\frac{D-2}{12} m\left(m^{2}-1\right) \delta_{m,-n} .}
\end{aligned}
$$

We see that in $D=2$ the string with dynamical geometry is anomaly free. That is the central charge in the Virasoro algebra equals zero.

Let us briefly comment this model. If one considers the ordinary bosonic string in two dimensions then one will find that the theory describes no continious degree of freedom because two Virasoro constraints and two gauge conditions eliminate both degrees of freedom $X^{0}$ and $X^{1}$. The situation in pure two-dimensional gravity with torsion is the same: one can always eliminate all continious degrees of freedom from the theory. At the same time the united theory of two-dimensional string with dynamical geometry describes two degrees of freedom and is anomaly free. The physical degrees of freedom in this case 
are the space component of the string $X^{1}$ and the conformal factor of the metric $g_{\alpha \beta}$. They yield positive definite Hamiltonian if the coupling constants in the Lagrangian satisfy (51).

At first sight the problem of tachyon will be successfully solved for two dimensional string with dynamical geometry. Indeed, in the ordinary approach the tachyon arises due to the constant $\alpha_{0}$ in the quantum expression for the constraint $L_{0}$. The negative value of $\alpha_{0}$ is intirely connected to the anomaly in the Virasoro algebra, for example, in the BRST approach. In our case the anomaly is absent and I see no reason for arising of the tachyon. This question as well as the spectrum and proper interpretation of the new Fock representation will be considered in detail elswhere.

\section{Conclusion}

Let us make a few remarks concerning construction of quantum gravity. General relativity as well as other theories with general coordinate invariance contains a set of nonlinear constraits. If one tries to quantize the theory in a perturbative approach then one finds that the algebra of the constraints do not closes in each order of the perturbation theory already at the classical level. This raises a question of consistency of the perturbative approach because to maintain general coordinate invariance at the quantum level one must retain all orders up to the order of the constraints. In four dimensional general relativity the constraints are nonpolinomial and therefore one must retain infinite number of perturbations to maintain the invariance at the quantum level. Therefore a nonperturbative approach to quantum gravity is desirable.

In the present paper we quantized two-dimensional gravity with torsion without any approximation. It is quite suprising that the algebra of nonpolinomial constraints do have a Fock space representation where the quantum algebra of the constraints coinsides with the classical Poisson bracket algebra. That is the theory is anomaly free neglecting the ghost contribution. To obtain a true anomaly free gauge theory one has to construct anomaly free representation for ghost fields. This representation appears to be different from the usual one and we hope that it exists.

To construct the representation of the constraints we have widely used the canonical transformations corresponding to extracting the longitidinal and transversal parts from the zweibein. This is a nontrivial problem in a curved background and the canonical transformation is nonlocal. The remarkable point is that the quadratic parts of the resulting constraints precisely coinside with the constraints of ordinary bosonic string. This interesting observation may be a common feature of gravity theories. Hopefully, a similar canonical transformation separating physical and unphysical modes exists in arbitrary dimensional space-time, and this is the proper way of approaching the problem of quantum gravity.

The approach used in the present paper demonstrates power and flexibility of the canonical transformations in separation of physical and unphysical modes in the theory. At the same time we have shown that even linear transformation of creation and annihilation operators can result in unitary inequivalent Fock space representations of the nonlinear operators. Nonlocality of the canonical transformation is not new and disastrous feature of the theory. For example, to seperate explicitly longitudinal part of the electromagnetic potential one also has to perform nonlocal transformation. There arises a question whether there exists and how to quantize the string with dynamical geome- 
try in terms of the initial variables in the Lagrangian. I hope a detailed elucidation of this problem in this relatively simple case will shed a new light on quantum canonical transformations and on the whole problem of quantum gravity.

The author would like to express his gratitude to I. V. Volovich for numerous enlightening discussions and to S. L. Lyakhovich, D. J. Schwarz and Th. Strobl for the conversations on Hamiltonian formulation of two-dimensional gravity with torsion and quantization.

\section{References}

[1] M. B. Green, J. H. Schwarz, and E. Witten. Superstring theory, volume 1,2. Cambridge U.P., Cambridge, 1987.

[2] L. Brink and M. Henneaux. Principles of String Theory. Plenum Press, New York and London, 1988.

[3] M. O. Katanaev and I. V. Volovich. String model with dynamical geometry and torsion. Phys. Lett., 175B(4):413-416, 1986.

[4] M. O. Katanaev and I. V. Volovich. Two-dimensional gravity with dynamical torsion and strings. Ann. Phys., 197(1):1-32, 1990.

[5] M. O. Katanaev and I. V. Volovich. Theory of defects in solids and three-dimensional gravity. Ann. Phys., 216(1):1-28, 1992.

[6] M. O. Katanaev. Complete integrability of two-dimensional gravity with dynamical torsion. J. Math. Phys., 31(4):882-891, 1990.

[7] W. Kummer and D. J. Schwarz. General analytic solution of $R^{2}$-gravity with dynamical torsion in two dimensions. Phys. Rev. D, 45(8):3628-3635, 1992.

[8] S. N. Solodukhin. Two-dimensional black holes with torsion. Preprint JINR E2-9333, page 10 pp., 1993.

[9] E. W. Mielke, F. Gronwald, Yu. N. Obukhov, R. Tresguerres, and F. W. Hehl. Towards complete integrability of two dimensional Poincaré gauge gravity. Preprint Cologne-thp-1993-H6, page 35 pp., 1993.

[10] K. J. Akdeniz, A. Kizilersü, and E. Rizaoğlu. Instanton and eigenmodes in a twodimensional theory of gravity with torsion. Phys. Lett., B215(1):81-83, 1988.

[11] M. O. Katanaev. Conformal invariance, extremals, and geodesics in two-dimensional gravity with torsion. J. Math. Phys., 32(9):2483-2496, 1991.

[12] M. O. Katanaev. All universal coverings of two-dimensional gravity with torsion. J. Math. Phys., 34(2):700-736, 1993.

[13] H. Grosse, W. Kummer, P. Prešnajder, and D. J. Schwarz. Novel symmetry of non-Einsteinian gravity in two dimensions. J. Math. Phys., 33:3892-3900, 1992. 
[14] T. Strobl. All symmetries of non-Einsteinian gravity in $d=2$. Int. J. Mod. Phys., A8:1383, 1993.

[15] M. O. Katanaev. String with dynamical geometry. Hamiltonian analysis in conformal gauge. Theor. Math. Phys., 80(2):239-252, 1989. [In Russian].

[16] K. J. Akdeniz, Ö. F. Dayi, and A. Kizilersü. Mod. Phys. Lett., A7:1757, 1992.

[17] R. Arnowitt, S. Deser, and S. W. Misner. The dynamics of general general relativity. In L. Witten, editor, Gravitation: an introduction to current research, New York London, 1962. John Wiley \& Sons, Inc.

[18] P. Schaller and T. Strobl. Canonical quantization of non-Einsteinian gravity and the problem of time. Preprint TUW-92-13, page 19 pp., 1992.

[19] W. Kummer and D. J. Schwarz. Renormalization of $R^{2}$-gravity with dynamical torsion in $d=2$. Nucl. Phys. B, 382:171-186, 1992.

[20] F. Haider and W. Kummer. Quantum functional integration of non-Einsteinian gravity in $d=2$. Preprint TUW-92-15, page 16 pp., 1992.

[21] N. Ikeda and K.-J. Izawa. Quantum gravity with dynamical torsion in two dimensions. Prog. Theor. Phys., 89:223, 1993.

[22] N. Ikeda and K.-J. Izawa. Gauge theory based on quadratic lie algebras and 2D gravity with dynamical torsion. Preprint RIMS-911, page 12pp., 1993.

[23] M. Henneaux, C. Teitelboim, and J. Zanelli. Gauge invariance and degree of freedom count. Nucl. Phys. B, 332(1):169-188, 1990.

[24] J.-L. Gervais and A. Neveu. Dual string spectrum in Polaykov's quantization. (II). Mode separation. Nucl. Phys., B209(1):125-145, 1982.

[25] E. Braaten, Th. Curtright, and C. Thorn. An exact operator solution of the quantum Liouville field theory. Ann. Phys., 147:365-416, 1983.

[26] R. Marnelius. Canonical quantization of Polyakov's string in arbitrary dimensions. Nucl. Phys., B211(1):14-28, 1983.

[27] H. J. Otto and G. Weigt. Construction of exponential Liouville field operators for closed string models. Z. Phys. C, 31(2):219-228, 1986. 